\title{
Inventory of benthic marine and estuarine algae and Cyanobacteria for Tabasco, México
}

\author{
Angela Catalina Mendoza-González $z^{*}$, Luz Elena Mateo-Cid ${ }^{1}$ \& Deisy Yazmin García- López ${ }^{1}$ \\ ${ }^{1}$ Instituto Politécnico Nacional, Escuela Nacional de Ciencias Biológicas, Departamento de Botánica. \\ Carpio y Plan de Ayala s/n, Campus Santo Tomas, C. P 11340, Ciudad de México, México. \\ *Corresponding author: E-mail: am7124@gmail.com
}

\begin{abstract}
MENDOZA-GONZÁLEZ, A. C., MATEO-CID, L. E., GARCÍA-LÓPEZ, D. Y. Inventory of benthic marine and estuarine algae and Cyanobacteria for Tabasco, México. Biota Neotropica. 17(4): e20170379. http://dx.doi. org/10.1590/1676-0611-BN-2017-0379
\end{abstract}

\begin{abstract}
Studies of benthic marine and estuarine algae as well as Cyanobacteria are in their early stages in the littoral of the state of Tabasco. Sixty seven taxa of seaweeds are currently known from Tabasco. The inventory of these organisms was made based on samples obtained in six localities, three of them in marine and three in estuarine environments. We found 147 species and subspecific taxa with Rhodophyta having the greatest number with 84 , followed by Chlorophyta 44, and finally Phaeophyceae 19. In addition, the 26 species of Cyanobacteria collected are recorded for the first time for the coast of Tabasco since there are no previous reports. The presence of 115 species of benthic marine and estuarine algae is reported for the first time for Tabasco. Most of the new records are Rhodophyta (53.9\%), followed by Chlorophyta (29.5\%) and Phaeophyceae (16.6\%). Of the 115 new records of algae for Tabasco, Gayliella fimbriata and Grateloupia subpectinata are new records also for the Mexican Atlantic. The highest number of species was recorded for Sanchez Magallanes breakwater, while the lowest number of taxa was recorded for Mecoacan Lagoon. According to the Feldmann and Cheney indexes the algal flora of the coast of Tabasco is tropical. The greatest diversity was found during the rainy season.
\end{abstract}

Keywords: Tabasco, Mexico, new records, marine, estuarine, algae, Cyanobacteria.

\section{Inventário de algas bentônicas marinhas e estuarinas e cianobactérias para Tabasco, México}

Resumo: Estudos sobre algas marinhas, estuarinas e cianobactérias para o litoral do estado de Tabasco encontram-se apenas nos estágios iniciais. Neste estudo, um inventário desses organismos foi feito com base em amostras obtidas em seis localidades, três delas marinhas e três em ambientes estuarinos. Os resultados indicaram a presença de 147 táxons, com Rhodophyta apresentando maior diversidade (84), seguido de Chlorophyta (44) e, finalmente, Phaeophyceae (19). Além disso, 26 espécies de Cyanobacteria são registradas pela primeira vez para a costa de Tabasco. A presença de 115 espécies de algas marinhas e estuarinas bentônicas é relatada peloa primeira vez para Tabasco. A maioria dos novos registros são de Rhodophyta (53.9\%), seguido por Chlorophyta (25.9\%), e Phaeophyceae (16.6\%). Dos 115 novos registros de algas para Tabasco, Gayliella fimbriata e Grateloupia subpectinata são novos registros para a costa atlântica do México. O maior número de espécies foi localizado no paredão de Sanchez Magallanes, enquanto o menos número de táxons foi localizado na Lagoa Mecoacán. De acordo com os índices de Feldmann e Cheney, a flora de algas da costa de Tabasco é tropical, e a maior diversidade foi encontrada durante a estação chuvosa.

Palavras-chave: Tabasco, México, registros novos, marinhos, estuarinos, algas, cianobactérias.

\section{Introduction}

The Gulf of México is characterized by a relatively shallow but well developed continental shelf with an extensive system of generally deep, hard banks of varying origin and composition (Fredericq et al. 2009). In spite of the extension of the Gulf of Mexico coast, in the Mexican portion it has been scarcely studied from the phycological point of view. The phycological literature (Ortega et al. 2001; Wynne 2017) highlights the limited records for the coast of Tabasco. Orozco-Vega \& Dreckmann (1995) reported six taxa for the Laguna Mecoacan, an estuarine environment; and Ramirez (1996) recorded 24 species of red algae (Rhodophyta) for the Sanchez-Magallanes breakwater. On the other hand, Dreckmann \& De Lara (2000) described the vegetative and reproductive structures of Gracilaria caudata (Rhodophyta) for Laguna Mecoacan. Sentíes \& Dreckmann (2013) updated information about the marine and estuarine algae for Tabasco, and recorded 50 taxa of algae for the Tabasco littoral. Recently, Quiroz-González et al. (2017) reported 17 Chlorophyta as new records for this coast. Of the five publications mentioned above, the most complete is that of Senties \& Dreckmann (2015), who published an inventory of marine and estuarine algae recorded until 2000 for the coast of Tabasco; also sampled in four localities: Playa El Bellote, Playa El Cangrejo, Laguna Mecoacan and 
Sánchez Magallanes breakwater, recording 50 taxa of algae, of which Rhodophyta had the highest number (38), followed by Phaeophyceae (7) and Chlorophyta (5). Quiroz-González et al (2017) carried out several collections in seven localities: Playa Miramar, Pico de Oro, Playa Paraiso, Poblado Chiltepec, Playa de Dos Bocas, Playa Las Brisas and Sanchez Magallanes breakwater; their study was based exclusively on Chlorophyta and the authors reported 17 new records of this group for the coast of Tabasco. In conclusion, it is evident that the length of the coast of Tabasco has not been equally inventoried and there are even portions which have not been studied at all, and the different groups of marine and estuarine algae have not been equally studied either.

Collectively, our knowledge about the cyanobacteria of the Tabasco coast is also limited. Cyanobacteria have been omitted in most studies conducted on the Mexican Atlantic benthic algae. Among the few reports that have been published is that of Mateo-Cid et al. (2013).

The aims of this study are to provide an updated revision of the diversity and distribution of the benthic marine and estuarine algae and Cyanobacteria of three new localities of the coast of Tabasco (San Pedro Centla, Laguna La Machona, La Machona), besides Sánchez Magallanes breakwater, Playa Paraíso breakwater and Laguna Mecoacan. We include records of new algae and cyanobacteria for Tabasco, as well as data about their distribution and temporal variation during the dry, rainy summer and rainy winter seasons (nortes). Using the Feldmann and Cheney indexes, the phycoflora of the coast of Tabasco will be classified as a function of the latitudinal gradient, and thus establish the basis for future taxonomic, ecological and biogeographic studies.

\section{Materials and methods}

\section{Study site and collections}

The shoreline of the state of Tabasco is between $092^{\circ} 28^{\text {' }}$ and $094^{\circ} 10^{\prime} \mathrm{W}$, and $17^{\circ} 15^{\prime}$ and $18^{\circ} 39^{\prime} \mathrm{N}$, located in the southeast meso-region of Mexico, bounded on the north by the Gulf of Mexico (Figure 1); the south by the coastline of Tabasco; to the east northeast by the state of Campeche; and to the west by the state of Veracruz (Hernández-Santana et al., 2008). The Tabasco coastline presents a morphological predominance of low sandy beaches. The coast of Tabasco has a warm humid weather type Am (f): one with a higher annual average temperature of $22^{\circ} \mathrm{C}$. Rains occur in the summer months and the precipitation of the driest month is less than $60 \mathrm{~mm}$; the percentage of winter rain is higher than $10.2 \%$ of the annual total (García y Vidal-Zepeda, 1990a), with minimum extreme values between 20 and $22^{\circ} \mathrm{C}$ in January, and maximum between 30 and $34^{\circ} \mathrm{C}$ in May (García y Vidal-Zepeda, 1990b). Sampling was carried out in the littoral of Tabasco in August and November 2015 and in April and June 2016. The collections were made in the intertidal zone, 0.5 to 1.5 meters deep for sampling sites were chosen by the type of substrate and of access to the localities. A total of 120 samples was collected at six localities: Sanchez Magallanes breakwater (marine environment), La Machona (marine environment), Playa Paraiso breakwater (marine environment), Laguna La Machona, Laguna Mecoacan and Pedro Centla (these three localities of estuarine environment) (Figure 1). Data on the GPS coordinates, type of substrate, depth and date of collection in which organisms were obtained are shown in Table 1. Algae were preserved in a $5 \%$ formalin/sea water solution. Small fragments were cut manually with a double-edged blade, and stained with aniline blue and hematoxilin-eosine for anatomical observations and measurements. All specimens of red and brown macroalgae were carefully checked under the microscope for epiphytes. Using a stereoscopic microscope Zeigen HG571405 and an optical microscope OLYMPUS CX3 epiphytic algae were found growing on macroalgae as Gracilaria, Grateloupia, Cladophora and Chnoospora, among others. Thalli were thoroughly reviewed from the base to the apical portion locating filamentous epiphytes and articulated or fleshy macrophytes. After that using a double- edged blade, longitudinal scrapings of $2 \mathrm{~cm}$ of each thallus were performed in order to locate microscopic algae (Mateo-Cid et al., 2013).

Semi-permanent slides were prepared using corn syrup/water 1:1 with a trace of phenol added to prevent fungal growth. The identification at the

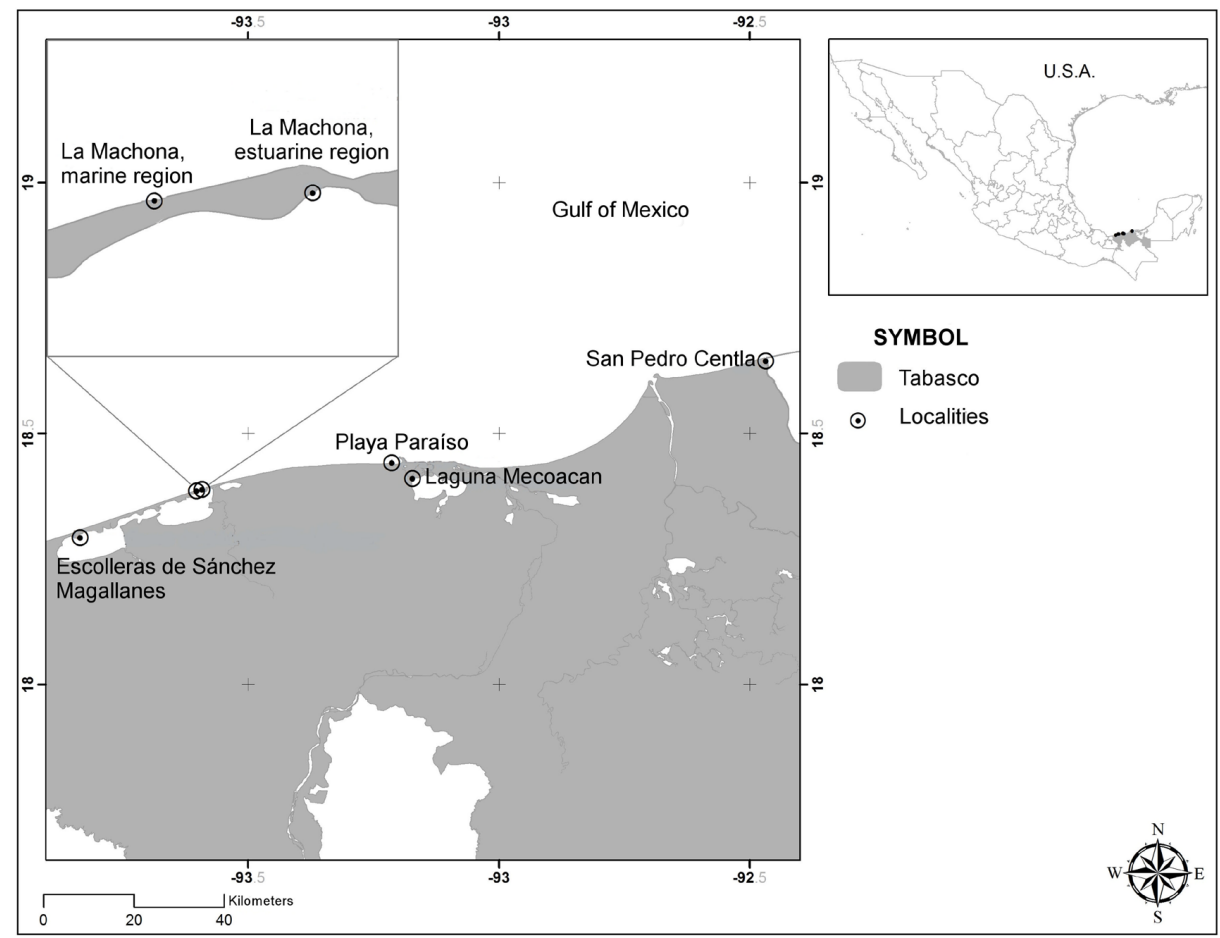

Figure 1. Map of the study area and sampling stations. 
Table 1. Location of sampling sites, substrate, environment, depth and collection dates.

\begin{tabular}{|c|c|c|c|c|c|}
\hline Locality & GPS Coordinates & Substrate & Environment & $\begin{array}{l}\text { Depth of } \\
\text { collection }\end{array}$ & Collection dates \\
\hline $\begin{array}{l}\text { 1. Sanchez Magallanes } \\
\text { breakwater }\end{array}$ & $18^{\circ} 17^{\prime} 26^{\prime \prime} \mathrm{N}, 93^{\circ} 50^{\prime} 03^{\prime \prime} \mathrm{W}$ & $\begin{array}{l}\text { Rocks and artificial } \\
\text { substrates }\end{array}$ & Marine & $0-1 \mathrm{~m}$ & August and November, 2015; April and June 2016 \\
\hline 2. La Machona & $18^{\circ} 38^{\prime} 51^{\prime \prime} \mathrm{N}, 93^{\circ} 60^{\prime} 61^{\prime \prime} \mathrm{W}$ & Rocks & Marine & $0 \mathrm{~m}$ & November 2015, April and June 2016 \\
\hline 3. Laguna La Machona & $18^{\circ} 17^{\prime} 56^{\prime \prime} \mathrm{N}, 93^{\circ} 51^{\prime} 03^{\prime \prime} \mathrm{W}$ & $\begin{array}{l}\text { Aquatic vascular plants } \\
\text { and artificial substrates }\end{array}$ & Estuarine & $0-1 \mathrm{~m}$ & November 2015, April and June 2016 \\
\hline 4. Playa Paraíso breakwater & $18^{\circ} 23^{\prime} 33^{\prime \prime} \mathrm{N}, 93^{\circ} 12^{\prime} 52^{\prime \prime} \mathrm{W}$ & Rocks & Marine & $0 \mathrm{~m}$ & August and November, 2015; April and June 2016 \\
\hline 5. Laguna Mecoacan & $18^{\circ} 24^{\prime} 37^{\prime \prime} \mathrm{N}, 93^{\circ} 10^{\prime} 21^{\prime \prime} \mathrm{W}$ & $\begin{array}{c}\text { Rocks and artificial } \\
\text { substrates }\end{array}$ & Estuarine & $0-2 \mathrm{~m}$ & November 2015, April and June 2016 \\
\hline 6. San Pedro Centla & $18^{\circ} 38^{\prime} 41^{\prime \prime} \mathrm{N}, 92^{\circ} 28^{\prime} 07^{\prime \prime} \mathrm{W}$ & $\begin{array}{l}\text { Cement floor and } \\
\text { artificial substrates }\end{array}$ & Estuarine & $0-1 \mathrm{~m}$ & November 2015, April and June 2016 \\
\hline
\end{tabular}

specific level was based mainly on Taylor (1960), Schneider \& Searles (1991), Littler \& Littler (2000), Cho et al. (2008), Dawes \& Mathieson (2008) and Won et al. (2009). For the determination of Cyanobacteria Gomont (1892), Anagnostidis \& Komárek (1988), Komárek \& Anagnostidis (1995), and Komárek et al. (2014) were used. The sequence of the floristic list follows the order proposed by Komárek (2010) for Cyanobacteria, Wynne (2017) and Guiry \& Guiry (2017) for red, brown and green algae. Most of the material analyzed is deposited and available for study in the phycological section of "Herbario Escuela Nacional de Ciencias Biológicas at the Instituto Politécnico Nacional, Mexico".

For the study of descriptive phytogeography, Feldmann (1937) proposed the R / P index (number of Rhodophyta species divided by the number of Phaeophyceae species) useful for classifying the flora of a given region as a function of the latitudinal gradient. Thus, a value of the ratio $\mathrm{R} / \mathrm{P}>4$ is characteristically found in tropical regions, while $\mathrm{R} / \mathrm{P}<2$ corresponds with a phycoflora of cool temperate regions. On the other hand, Cheney (1977) included the Chlorophyta (C) based on the one previously mentioned: $(\mathrm{R}+\mathrm{C}) / \mathrm{P}$ and verified that values of the ratio $(\mathrm{R}+\mathrm{C}) / \mathrm{P}>6$ are obtained in tropical floras, while those in temperate-cold seas are $<3$.

\section{Results}

\section{New records of algae and cyanobacteria for Tabasco}

A total of 173 taxa was identified, 84 of them are Rhodophyta, 44 Chlorophyta, 19 Phaeophyceae as well as 26 Cyanobacteria. The taxa identified from our collections are listed in Table 2 with sampling sites, seasonality, marine or estuarine environment and type of substrate where the organisms were collected, where the reference comes from, either from bibliography or collected by the authors, observations on new records for Tabasco and the Atlantic coast of Mexico and herbarium number. Many of the new algae records located in this study are epiphytes and they are not reported frequently in the floristic lists of Mexican coast of Gulf of Mexico (Ortega et al. 2001, Mateo-Cid et al. 2013, Senties \& Dreckmann 2013, Wynne 2017).

Two red algae, Gayliella fimbriata (Setchell \& N.L. Gardner) T.O. Cho \& S.M. Boo and Grateloupia subpectinata Holmes are new records for the Atlantic coast of Mexico. Twenty six Cyanobacteria, 62 Rhodophyta, 18 Phaeophyceae and 34 Chlorophyta are new records for Tabasco. These new records are indicated in Table 2. The following families were the best represented in number of species in the study area: Rhodomelaceae (14), Ceramiaceae (12), Gracilariaceae (7), Cladophoraceae (19), Bryopsidaceae (8) and Ulvaceae (7). These families collectively include $45.57 \%$ of the total floristic diversity registered. The highest species richness was recorded in sampling sites with rocky substrate. The locality with the greatest diversity of species and subspecific taxa corresponds to the Sanchez Magallanes breakwater with 123 species, followed by the Playa Paraíso breakwater with 75, Laguna La Machona 35, La Machona 31, San Pedro Centla 26 and finally Laguna Mecoacan with 20 species (Figure 2).

Species and subspecific taxa such as Jania ungulata f. brevior Yendo, Centroceras gasparrinii (Meneghini) Kützing, Spyridia clavata Kützing, Polysiphonia scopolorum var. villum (J. Agardh) Hollenberg, Peyssonnelia armorica (P.L. Crouan \& H.M. Crouan) Weber-van Bosse, Hecatonema floridanum (W.R. Taylor) W.R. Taylor, Herponema tortugense (W.R. Taylor) W.R. Taylor, Rosenvingea orientalis (J. Agardh) Børgesen, Blidingia minima (Nägeli ex Kützing) Kylin, Cladophora crispula Vickers and Pseudobryopsis blomquistii Diaz-Piferrer are considered unusual because few records are in the floristic lists of the Mexican coast of the Gulf of Mexico (Ortega et al. 2001, Mateo-Cid et al. 2013, Senties \& Dreckmann 2013, Wynne 2017).

Thirty of the 67 species previously recorded for Tabasco (Orozco-Vega \& Dreckmann 1995, Ramirez 1996, Dreckmann \& De Lara-Isassi 2000, Senties \& Dreckmann 2013, Quiroz-González et al., 2017) were found in this study. The other 115 species (table 2) are considered new records for Tabasco.

\section{New records for the Atlantic coast of Mexico}

Gayliella fimbriata and Grateloupia subpectinata of the coast of Tabasco, Mexico were recorded for the first time.

\section{Description}

Gayliella fimbriata (Setchell \& N.L. Gardner) T.O. Cho \& S.M. Boo (Figures 3a-d)

Type locality: Eureka, near La Paz, Lower California Mexico (Setchell \& Gardner 1924: 777).

Basionym: Ceramium fimbriatum Setchell \& N.L. Gardner 1924

Representative specimens examined: Sánchez Magallanes (Mendoza González \& García López, 26.viii.2015, ENCB 22091, Vegetative).

Habit and anatomy: Thalli are $0.5-1.0 \mathrm{~cm}$ high, consisting of prostrate axes giving rise to erect axes (Figure 2). Erect axes bear forcipate incurved and complanate apical regions, main filaments $85-95 \mu \mathrm{m}$ diameter at the nodes. Axial cells are spherical to cylindrical. The acropetal corticating filaments are 3-4 cells long, while the basipetal ones are 2-3 cells long. Branches are regularly alternate. Gland cells usually develop from cortical cells of acropetally and rarely basipetally corticating filaments, becoming strongly protruding, and are clavate, $60-65 \mu \mathrm{m} \times 30-34 \mu \mathrm{m}$.

Reproductive thalli were not found in our collections.

Comments: A particularly interesting case is the finding of Gayliella fimbriata, because this species was first described by Setchell and Gardner (1924) for the Bay of La Paz (Gulf of California, Mexico) as Ceramium fimbriatum and was subsequently transferred by Cho et al. (2008) to G. fimbriata. The presence of characteristic gland cells on the cortical cells and the dimensions of thalli agree with those recorded in specimens from La Paz, BCS, Mexico (Setchell \& Gardner, 1924, Dawson, 1962). 
Table 2. Marine and estuarine algae and Cyanobacteria of the coast of Tabasco (The abbreviations are explained at the end of the table).

\section{DIVISION/SPECIES \\ LOCALITIES SEASONALITY ENVIRONMENT SUBSTRATE REFERENCE OBS}

ENCB Herbarium number

\section{CYANOBACTERIA \\ Cyanophyceae \\ Oscillatoriales \\ Oscillatoriaceae}

1. Blennothrix lyngbyacea (Kützing ex Gomont) Anagnostidis et Komárek

2. Lyngbya confervoides C. Agardh ex Gomont

3. L. majuscula Harvey ex Gomont

4. L. salina Kützing ex Gomont

5. L. semiplena J. Agardh ex Gomont

\section{Homoeotrichaceae}

6. Ammatoidea aegaea

Anagnostidis \& Pantazidou

$\begin{array}{ccc}1,6 & \text { W, S, R } & \text { M,E } \\ 1,4,6 & \text { W,S, R } & \text { M,E } \\ 1,3 & \text { R } & \text { M,E } \\ 1 & \text { W, R } & \text { M } \\ 1,6 & \text { W, R } & \text { M,E }\end{array}$

Epi, As
As
Epi, Avp
Epi
Epi

A

NRT

21883

hormidiaceae

7. Coleofasciculus chthonoplastes

1

$\mathrm{R}$

M

Epi

A

NRT 217692196321992

(Thuret ex Gomont) M. Siegesmund, J.R. Johansen \& T. Friedl

\section{Nostocales}

Aphanizomenonaceae

8. Nodularia harveyana Thuret ex Bornet \& Flahault

NRT

2193721962

21968

NRT

2201022014

Rivulariaceae

9. Calothrix confervicola C. Agardh ex Bornet \& Flahault

10. C. parietina Thuret ex

Bornet \& Flahault

Scytonemataceae

11. Scytonematopsis crustacea (Thuret ex Bornet \& Flahault) Koválik \& Komárek

Pseudanabaenales

Pseudanabaenaceae

12. Leptolyngbya marina (N.L. Gardner) Anagnostidis

$1,2,3,4,6$

W, R

M,E

Epi

A NRT

2187522111

Chroococcales

Chroococcaceae

13. Chroococcus ercegovicii Komárek \& Anagnostidis

14. C. turgidus (Kützing) Nägeli

15. Pseudocapsa maritima Komárek

Pleurocapsales

Dermocarpellaceae

16. Cyanocystis hemisphaerica

(Setchell \& N.L. Gardner) Kaas

17. Stanieria sublitoralis (A. Lindstedt)

Anagnostidis \& Pantazidou

$\begin{array}{cc}6 & \text { W } \\ 1 & \text { S, R } \\ 6 & \text { W }\end{array}$

\section{E}

Epi

A NRT

22173

M

Epi

A NRT

22014

E

Epi

A NRT

22173

Entophysalidaceae

18. Entophysalis conferta (Kützing) F.E. Drouet \& W.A. Daily

\begin{tabular}{|c|c|c|c|c|c|c|}
\hline 1 & $\mathrm{R}$ & $\mathrm{M}$ & Epi & A & NRT & 21991 \\
\hline 1,2 & $\mathrm{~S}, \mathrm{R}$ & M & Epi & A & NRT & 21921 \\
\hline $1,2,3,4,6$ & $\mathrm{~W}, \mathrm{~S}, \mathrm{R}$ & $\mathrm{M}, \mathrm{E}$ & Epi & A & NRT & 2177221922 \\
\hline 3,5 & W & $\mathrm{E}$ & Epi & A & NRT & 22160 \\
\hline 1 & $\mathrm{R}$ & M & Epi & A & NRT & 22311 \\
\hline 1 & $\mathrm{~W}, \mathrm{R}$ & M & Epi & $\mathrm{A}$ & NRT & 21906 \\
\hline 5 & W & $\mathrm{E}$ & Epi & A & NRT & 21995 \\
\hline
\end{tabular}

19. Xenococeae

Setchell \& N.L. Gardner

20. X. minimus Geitler

21. X. gilkeyae Setchell \& N.L .Gardner

22. X. pyriformis Setchell \& N.L. Gardner

$\mathrm{W}$

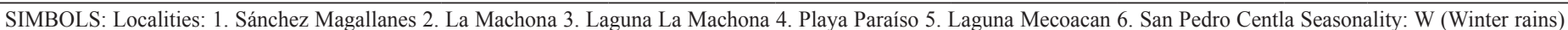

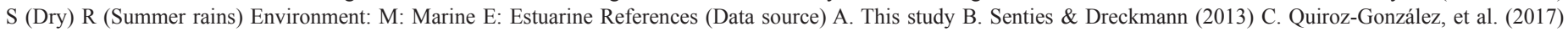

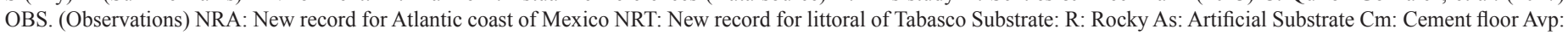
Aquatic vascular plants Epi: Epiphytic on other algae 
Table 2. Continued...

\begin{tabular}{|c|c|c|c|c|c|c|c|}
\hline DIVISION/SPECIES & LOCALITIES & SEASONALITY & ENVIRONMENT & SUBSTRATE & REFERENCE & OBS. & $\begin{array}{c}\text { ENCB Herbarium } \\
\text { number }\end{array}$ \\
\hline \multicolumn{8}{|l|}{ Synechococcales } \\
\hline \multicolumn{8}{|l|}{ Chamaesiphonaceae } \\
\hline 23. Chamaesiphon rostafinskii Hansgirg & 1 & $\mathrm{R}$ & M & Epi & A & NRT & 22111 \\
\hline \multicolumn{8}{|l|}{ Merismopediaceae } \\
\hline 24. Aphanocapsa litoralis Hansgirg & 1 & $\mathrm{~S}, \mathrm{R}$ & M & Epi & A & NRT & 22152 \\
\hline 25. A. marina Hansgirg & 6 & $\mathrm{R}$ & $\mathrm{E}$ & Epi & A & NRT & 22117 \\
\hline 26. Synechocystis minuscula Woronichin & 1,6 & $\mathrm{~W}, \mathrm{R}$ & $\mathrm{M}, \mathrm{E}$ & Epi & A & NRT & 21998 \\
\hline \multicolumn{8}{|l|}{ RHODOPHYTA } \\
\hline \multicolumn{8}{|l|}{ Bangiophyceae } \\
\hline Bangiales & & & & & & & 21906 \\
\hline Bangiaceae & & & & & & & 2199622133 \\
\hline $\begin{array}{l}\text { 27. Bangia atropurpurea (Mertens ex } \\
\text { Roth) C. Agardh }\end{array}$ & $1,3,5$ & $\mathrm{~W}, \mathrm{R}$ & $\mathrm{M}, \mathrm{E}$ & As, $\mathrm{R}$ & A & NRT & \\
\hline 28. B. fuscopurpurea (Dillwyn) Lyngbye & 6 & $\mathrm{~W}, \mathrm{~S}$ & $\mathrm{E}$ & As & A & NRT & 217662184822071 \\
\hline \multicolumn{8}{|l|}{ Compsopogonophyceae } \\
\hline \multicolumn{8}{|l|}{ Erythropeltales } \\
\hline \multicolumn{8}{|l|}{ Erythrotrichiaceae } \\
\hline $\begin{array}{l}\text { 29. Erythrotrichia carnea } \\
\text { (Dillwyn) J. Agardh }\end{array}$ & $1,2,3,4,5$ & $\mathrm{~W}, \mathrm{R}$ & $\mathrm{M}, \mathrm{E}$ & Epi & $\mathrm{A}, \mathrm{B}$ & & 21770 \\
\hline $\begin{array}{l}\text { 30. Sahlingia subintegra (Rosenvinge) } \\
\text { Kornmann }\end{array}$ & 2,4 & $\mathrm{~S}, \mathrm{R}$ & M & Epi & A & NRT & 2191322072 \\
\hline \multicolumn{8}{|l|}{ Stylonematophyceae } \\
\hline \multicolumn{8}{|l|}{ Stylonematales } \\
\hline \multicolumn{8}{|l|}{ Stylonemataceae } \\
\hline $\begin{array}{l}\text { 31. Chroodactylon ornatum (C. Agardh) } \\
\text { Basson }\end{array}$ & 1,4 & $\mathrm{R}$ & M & Epi & A & NRT & 21970 \\
\hline $\begin{array}{l}\text { 32. Stylonema alsidii (Zanardini) K.M. } \\
\text { Drew }\end{array}$ & 1,4 & $\mathrm{~S}, \mathrm{R}$ & M & Epi & A & NRT & 2187522062 \\
\hline \multicolumn{8}{|l|}{ Florideophyceae } \\
\hline \multicolumn{8}{|l|}{ Acrochaetiales } \\
\hline \multicolumn{8}{|l|}{ Acrochaetiaceae } \\
\hline 33. Acrochaetium flexuosum Vickers & 1,3 & $\mathrm{~W}, \mathrm{R}$ & $\mathrm{M}, \mathrm{E}$ & Epi & A & NRT & 2176622063 \\
\hline $\begin{array}{l}\text { 34. A. microscopicum (Nägeli ex Kützing) } \\
\text { Nägeli }\end{array}$ & $1,2,4,6$ & $\mathrm{~W}, \mathrm{~S}, \mathrm{R}$ & $\mathrm{M}, \mathrm{E}$ & Epi & A & NRT & 217722184922072 \\
\hline 35. Acrochaetium sancti-thomae Børgesen & 1 & $\mathrm{~S}$ & M & Epi & A & NRT & 22093 \\
\hline 36. A. sagraeanum (Montagne) Bornet & 4 & $\mathrm{~S}$ & M & Epi & A & NRT & TAB-ENCB/A2 \\
\hline 37. A. secundatum (Lyngbye) Nägeli & 4 & $\mathrm{~S}$ & M & Epi & A & NRT & TAB-17/A1 \\
\hline \multicolumn{8}{|l|}{ Colaconematales } \\
\hline \multicolumn{8}{|l|}{ Coleconemataceae } \\
\hline $\begin{array}{l}\text { 38. Colaconema dasyae (Collins) } \\
\text { Stegenga, I. Mol, Prud'homme van } \\
\text { Reine \& Lokhorst }\end{array}$ & 1 & $\mathrm{R}$ & M & Epi & A & NRT & 2194822064 \\
\hline 39. C. daviesii (Dillwyn) Stegenga & 1 & $\mathrm{~S}$ & M & Epi & A & NRT & 22152 \\
\hline $\begin{array}{l}\text { 40. C. hallandicum (Kylin) Afonso-Carillo, } \\
\text { Sanson, Sangil \& Diaz-Villa }\end{array}$ & 3 & $\mathrm{R}$ & $\mathrm{E}$ & As & A & NRT & TAB-ENCB-C3 \\
\hline $\begin{array}{l}\text { 41. C. hypneae (Børgesen) A.A. } \\
\text { Santos \& W.N. Moura }\end{array}$ & $1,2,3,4$ & $\mathrm{~S}, \mathrm{R}$ & $\mathrm{M}, \mathrm{E}$ & Epi & A & NRT & $\begin{array}{c}217692189821913 \\
2208221934\end{array}$ \\
\hline 42. C. savianum (Meneghini) R. Nielsen & 5 & $\mathrm{~W}$ & $\mathrm{E}$ & Epi & A & NRT & 21995 \\
\hline \multicolumn{8}{|l|}{ Corallinales } \\
\hline \multicolumn{8}{|l|}{ Corallinaceae } \\
\hline $\begin{array}{l}\text { 43. Hydrolithon farinosum } \\
\text { (J.V. Lamouroux) D.L. } \\
\text { Penrose \& Y.M. Chamberlain }\end{array}$ & 4 & $\mathrm{~S}, \mathrm{R}$ & M & Epi & A & NRT & 21922 \\
\hline 44. Jania adhaerens J.V. Lamouroux & 1 & $\mathrm{R}$ & M & $\mathrm{R}$ & A & NRT & 22459 \\
\hline
\end{tabular}

SIMBOLS: Localities: 1. Sánchez Magallanes 2. La Machona 3. Laguna La Machona 4. Playa Paraíso 5. Laguna Mecoacan 6. San Pedro Centla Seasonality: W (Winter rains) S (Dry) R (Summer rains) Environment: M: Marine E: Estuarine References (Data source) A. This study B. Senties \& Dreckmann (2013) C. Quiroz-González, et al. (2017) OBS. (Observations) NRA: New record for Atlantic coast of Mexico NRT: New record for littoral of Tabasco Substrate: R: Rocky As: Artificial Substrate Cm: Cement floor Avp: Aquatic vascular plants Epi: Epiphytic on other algae 
Table 2. Continued..

\begin{tabular}{|c|c|c|c|c|c|c|c|}
\hline DIVISION/SPECIES & LOCALITIES & SEASONALITY & ENVIRONMENT & SUBSTRATE & REFERENCE & OBS. & $\begin{array}{c}\text { ENCB Herbarium } \\
\text { number }\end{array}$ \\
\hline 45. J. capillacea Harvey & 4 & $\mathrm{R}$ & $\mathrm{M}$ & $\mathrm{R}$ & A & NRT & 22457 \\
\hline 46. J. ungulata f. brevior Yendo & 4 & $\mathrm{R}$ & M & Epi & A & NRT & 21206 \\
\hline 47. J. rubens (Linnaeus) J.V. Lamouroux & 4 & $\mathrm{~S}, \mathrm{R}$ & M & $\mathrm{R}$ & A & NRT & 22458 \\
\hline $\begin{array}{l}\text { 48. Lithophyllum prototypum (Foslie) } \\
\text { Foslie }\end{array}$ & 4 & $\mathrm{~S}$ & M & $\mathrm{R}$ & A & NRT & 21621 \\
\hline 49. L. stictiforme (Areschoug) Hauck & 4 & $\mathrm{~S}, \mathrm{R}$ & M & $\mathrm{R}$ & A & NRT & 21620 \\
\hline \multicolumn{8}{|l|}{ Ceramiales } \\
\hline \multicolumn{8}{|l|}{ Callithamniaceae } \\
\hline $\begin{array}{l}\text { 50. Aglaothamnion boergesenii (Aponte \& } \\
\text { D.L. Ballantine) L'Hardy-Halos \& Rueness }\end{array}$ & 1 & $\mathrm{R}$ & M & $\mathrm{R}$ & A & NRT & TAB-15-49/06 \\
\hline $\begin{array}{l}\text { 51. Callithamnion corymbosum } \\
\text { (J.E. Smith) Lyngbye }\end{array}$ & 1 & $\mathrm{~S}, \mathrm{R}$ & M & Epi, $\mathrm{R}$ & A, B & & 2201122042 \\
\hline \multicolumn{8}{|l|}{ Ceramiaceae } \\
\hline $\begin{array}{l}\text { 52. Antithamnionella boergesenii } \\
\text { (Cormaci \& G.Furnari) Athanasiadis }\end{array}$ & 1 & $\mathrm{R}$ & M & Epi & A & NRT & 22088 \\
\hline $\begin{array}{l}\text { 53. An. elegans (Berthold) } \\
\text { J.H. Price \& D.M. John }\end{array}$ & 1 & $\mathrm{R}$ & M & Epi & A & NRT & 2192922042 \\
\hline $\begin{array}{l}\text { 54. Callithamniella tingitana } \\
\text { (Schousboe ex Bornet) Feldmann-Mazoyer }\end{array}$ & 1 & $\mathrm{R}$ & M & Epi & A & NRT & 22138 \\
\hline $\begin{array}{l}\text { 55. Centroceras gasparrinii (Meneghini) } \\
\text { Kützing }\end{array}$ & 1,4 & $\mathrm{~S}, \mathrm{R}$ & M & $\mathrm{R}, \mathrm{Cm}$ & A & NRT & 220432204422045 \\
\hline 56. Cn. hyalacanthum Kützing & 1,4 & $\mathrm{R}$ & M & Epi & A & NRT & 2187422047 \\
\hline 57. Cn. micracanthum Kützing & 1,4 & $\mathrm{~W}, \mathrm{~S}, \mathrm{R}$ & M & $\mathrm{R}$ & A & NRT & 22039 \\
\hline $\begin{array}{l}\text { 58. Ceramium brevizonatum var. } \\
\text { caraibicum } \text { H.E. Petersen \& Børgesen }\end{array}$ & 4 & $\mathrm{R}$ & M & $\mathrm{R}$ & A & NRT & 22038 \\
\hline 59. C. cruciatum F.S. Collins \& Hervey & 1,4 & $\mathrm{R}$ & M & $\mathrm{R}$ & $\mathrm{A}$ & NRT & 2188322056 \\
\hline 60. C. leutzelburgii Schmidt & 5 & $\mathrm{~S}, \mathrm{R}$ & $\mathrm{E}$ & Epi & A & NRT & 22040 \\
\hline $\begin{array}{l}\text { 61. Gayliella flaccida (Harvey) T. O. Cho } \\
\text { \& L. J. McIvor }\end{array}$ & 1,4 & $\mathrm{~S}, \mathrm{R}$ & M & Epi & A & NRT & 2187422037 \\
\hline $\begin{array}{l}\text { 62. G. fimbriata (Setchell \& N.L. Gardner) } \\
\text { T.O. Cho \& S.M. Boo }\end{array}$ & 1 & $\mathrm{R}$ & M & Epi & A & NRA & 22091 \\
\hline $\begin{array}{l}\text { 63. G. mazoyerae T.O. Cho, } \\
\text { Fredericq \& Hommersand }\end{array}$ & 1 & $\mathrm{R}$ & M & Epi & A & NRT & 22025 \\
\hline $\begin{array}{l}\text { 64. G. transversalis (Collins \& Hervey) } \\
\text { T.O. Cho \& Fredericq }\end{array}$ & 1 & $\mathrm{R}$ & M & Epi & A & NRT & 22111 \\
\hline \multicolumn{8}{|l|}{ Spyridiaceae } \\
\hline 65. Spyridia clavata Kützing & 1 & $\mathrm{~S}$ & M & $\mathrm{R}$ & A & NRT & 2197721978 \\
\hline $\begin{array}{l}\text { 66. S. hypnoides (Bory de Saint-Vincent) } \\
\text { Papenfuss }\end{array}$ & 1 & $\mathrm{~S}$ & M & $\mathrm{R}$ & A, B & & 2209022091 \\
\hline \multicolumn{8}{|l|}{ Wrangeliaceae } \\
\hline 67. Wrangelia argus (Montagne) Montagne & $1,2,4$ & $\mathrm{~W}, \mathrm{~S}, \mathrm{R}$ & M & Epi, R, As & A & NRT & 219732197421975 \\
\hline \multicolumn{8}{|l|}{ Dasyaceae } \\
\hline $\begin{array}{l}\text { 68. Dasya pedicellata (C. Agardh) C. } \\
\text { Agardh }\end{array}$ & 5 & $\mathrm{~S}$ & $\mathrm{E}$ & $\mathrm{Cm}$ & A, B & & 22034 \\
\hline $\begin{array}{l}\text { 69. Heterosiphonia crispella (C. Agardh) } \\
\text { M.J. Wynne }\end{array}$ & 4 & $\mathrm{R}$ & M & Epi & A & NRT & 22009 \\
\hline \multicolumn{8}{|l|}{ Delesseriaceae } \\
\hline $\begin{array}{l}\text { 70. Caloglossa leprieurii (Montagne) G. } \\
\text { Martens }\end{array}$ & 6 & $\mathrm{~W}, \mathrm{~S}$ & $\mathrm{E}$ & $\mathrm{Cm}$ & A & NRT & 22075 \\
\hline \multicolumn{8}{|l|}{ Rhodomelaceae } \\
\hline $\begin{array}{l}\text { 71. Acanthophora muscoides (Linnaeus) } \\
\text { Bory }\end{array}$ & 1 & W & M & $\mathrm{R}$ & A & NRT & 21767 \\
\hline 72. A. spicifera (Vahl) Børgesen & $1,2,3,4$ & $\mathrm{~W}, \mathrm{~S}, \mathrm{R}$ & $\mathrm{M}, \mathrm{E}$ & $\mathrm{R}$ & $\mathrm{A}, \mathrm{B}$ & & 217692199922000 \\
\hline $\begin{array}{l}\text { 73. Bostrychia radicans (Montagne) } \\
\text { Montagne }\end{array}$ & 6 & $\mathrm{~S}$ & $\mathrm{E}$ & As & A & NRT & TAB-15-D/11 \\
\hline
\end{tabular}

SIMBOLS: Localities: 1. Sánchez Magallanes 2. La Machona 3. Laguna La Machona 4. Playa Paraíso 5. Laguna Mecoacan 6. San Pedro Centla Seasonality: W (Winter rains) S (Dry) R (Summer rains) Environment: M: Marine E: Estuarine References (Data source) A. This study B. Senties \& Dreckmann (2013) C. Quiroz-González, et al. (2017) OBS. (Observations) NRA: New record for Atlantic coast of Mexico NRT: New record for littoral of Tabasco Substrate: R: Rocky As: Artificial Substrate Cm: Cement floor Avp: Aquatic vascular plants Epi: Epiphytic on other algae 
Table 2. Continued...

\begin{tabular}{|c|c|c|c|c|c|c|c|}
\hline DIVISION/SPECIES & LOCALITIES & SEASONALITY & ENVIRONMENT & SUBSTRATE & REFERENCE & OBS. & $\begin{array}{c}\text { ENCB Herbarium } \\
\text { number }\end{array}$ \\
\hline $\begin{array}{l}\text { 74. Bryocladia cuspidata (J. Agardh) De } \\
\text { Toni }\end{array}$ & $1,2,4$ & $\mathrm{~W}, \mathrm{~S}, \mathrm{R}$ & M & $\mathrm{R}$ & $\mathrm{A}, \mathrm{B}$ & & 2199022013 \\
\hline 75. B. thyrsigera (J. Agardh) F. Schimtz & $1,2,3,4$ & $\mathrm{~S}, \mathrm{R}$ & $\mathrm{M}, \mathrm{E}$ & $\mathrm{R}$ & A & NRT & 219062201022012 \\
\hline 76. Chondria collinsiana M. Howe & 1 & $\mathrm{R}$ & M & $\mathrm{R}$ & A & NRT & 22140 \\
\hline $\begin{array}{l}\text { 77. Melanothamnus ferulaceus } \\
\text { (Suhr ex J. Agardh) Díaz-Tapia \& Maggs }\end{array}$ & 1,2 & $\mathrm{~S}, \mathrm{R}$ & M & R, Epi & A, B & & 2198121983 \\
\hline $\begin{array}{l}\text { 78. M. sphaerocarpus (Børgesen) Díaz- } \\
\text { Tapia \& Maggs }\end{array}$ & 4,5 & $\mathrm{~W}, \mathrm{R}$ & $\mathrm{M}, \mathrm{E}$ & As, Epi & A, B & & 2198022005 \\
\hline $\begin{array}{l}\text { 79. Polysiphonia atlantica Kapraun \& J.N. } \\
\text { Norris }\end{array}$ & 4,5 & $\mathrm{~W}, \mathrm{R}$ & $\mathrm{M}, \mathrm{E}$ & Epi & A & NRT & 2200522115 \\
\hline 80. P. havanensis Montagne & $1,4,6$ & $\mathrm{~W}, \mathrm{~S}, \mathrm{R}$ & $\mathrm{M}, \mathrm{E}$ & Epi, As & $\mathrm{A}, \mathrm{B}$ & & 220152211422117 \\
\hline $\begin{array}{l}\text { 81. P. schneideri B. Stuercke \& D.W. } \\
\text { Freshwater }\end{array}$ & 1 & $\mathrm{~W}$ & M & As & A & NRT & 22140 \\
\hline $\begin{array}{l}\text { 82. P. scopolorum var. villum (J. Agardh) } \\
\text { Hollenberg }\end{array}$ & 1 & $\mathrm{R}$ & M & Epi & A & NRT & 22147 \\
\hline 83. P. subtilissima Montagne & $1,4,5$ & $\mathrm{~W}, \mathrm{~S}, \mathrm{R}$ & $\mathrm{M}, \mathrm{E}$ & $\mathrm{Cm}, \mathrm{As}$ & $\mathrm{A}, \mathrm{B}$ & & 220022200322113 \\
\hline $\begin{array}{l}\text { 84. Yuzurua poiteauii var. gemmifera } \\
\text { (Harvey) M.J. Wynne }\end{array}$ & 3 & W & $\mathrm{E}$ & As & A & NRT & 22120 \\
\hline \multicolumn{8}{|l|}{ Gelidiales } \\
\hline \multicolumn{8}{|l|}{ Gelidiaceae } \\
\hline $\begin{array}{l}\text { 85. Gelidium americanum (W.R. Taylor) } \\
\text { Santelices }\end{array}$ & $2,4,5$ & $\mathrm{~W}, \mathrm{R}$ & $\mathrm{M}, \mathrm{E}$ & $\mathrm{Cm}, \mathrm{R}$ & A & NRT & 220232202522032 \\
\hline 86. G. corneum (Hudson) J.V. Lamouroux & 1,2 & $\mathrm{~W}$ & M & $\mathrm{R}$ & A & NRT & 2202622027 \\
\hline 87. G. pusillum (Stackhouse) Le Jolis & 1,4 & $\mathrm{~W}, \mathrm{~S}, \mathrm{R}$ & M & $\mathrm{R}$ & $\mathrm{A}, \mathrm{B}$ & & 2200722008 \\
\hline \multicolumn{8}{|l|}{ Pterocladiaceae } \\
\hline $\begin{array}{l}\text { 88. Pterocladiella sanctarum } \\
\text { (Feldmann \& Hamel) Santelices }\end{array}$ & 1 & W & M & $\mathrm{R}$ & A & NRT & 22061 \\
\hline \multicolumn{8}{|l|}{ Hildenbrandiales } \\
\hline \multicolumn{8}{|l|}{ Hildenbrandiaceae } \\
\hline $\begin{array}{l}\text { 89. Hildenbrandia rubra (Sommerfelt) } \\
\text { Meneghini }\end{array}$ & 1 & $\mathrm{R}$ & M & $\mathrm{R}$ & A & NRT & 21272 \\
\hline \multicolumn{8}{|l|}{ Peyssonneliales } \\
\hline \multicolumn{8}{|l|}{ Peyssonneliaceae } \\
\hline $\begin{array}{l}\text { 90. Peyssonnelia armorica (P.L. Crouan \& } \\
\text { H.M. Crouan) Weber-van Bosse }\end{array}$ & 4 & $\mathrm{R}$ & M & Epi & A & NRT & 21620 \\
\hline 91. P. rubra (Greville) J. Agardh & 1,4 & $\mathrm{R}$ & M & $\mathrm{R}$ & A & NRT & 21348 \\
\hline \multicolumn{8}{|l|}{ Gigartinales } \\
\hline \multicolumn{8}{|l|}{ Cystocloniaceae } \\
\hline 92. Hypnea cornuta (Kützing) J. Agardh & 1 & $\mathrm{R}$ & M & $\mathrm{R}$ & A & NRT & 22049 \\
\hline $\begin{array}{l}\text { 93. H. musciformis (Wulfen in Jacquin) J. V. } \\
\text { Lamouroux }\end{array}$ & $1,2,4$ & $\mathrm{~S}, \mathrm{R}$ & M & Epi, R, As & $\mathrm{A}, \mathrm{B}$ & & 220762207822081 \\
\hline 94. H. spinella (C. Agardh) Kützing & $1,2,3,4$ & $\mathrm{~W}, \mathrm{~S}, \mathrm{R}$ & $\mathrm{M}, \mathrm{E}$ & R, Epi & $\mathrm{A}, \mathrm{B}$ & & 218752205122052 \\
\hline 95. H. valentiae (Turner) Montagne & 1,2 & $\mathrm{~S}, \mathrm{R}$ & M & $\mathrm{R}$ & A & NRT & 22050 \\
\hline \multicolumn{8}{|l|}{ Gigartinaceae } \\
\hline $\begin{array}{l}\text { 96. Chondracanthus acicularis (Roth) } \\
\text { Fredericq }\end{array}$ & 1 & $\mathrm{~S}$ & M & $\mathrm{R}$ & A & NRT & 22108 \\
\hline \multicolumn{8}{|l|}{ Phyllophoraceae } \\
\hline $\begin{array}{l}\text { 97. Gymnogongrus griffithsiae (Turner) } \\
\text { Martius }\end{array}$ & 1 & $\mathrm{~W}$ & M & $\mathrm{R}$ & A & NRT & 22070 \\
\hline 98. G. tenuis J. Agardh & 1 & $\mathrm{~W}, \mathrm{R}$ & M & $\mathrm{R}$ & A & NRT & 2187622103 \\
\hline \multicolumn{8}{|l|}{ Gracilariales } \\
\hline \multicolumn{8}{|l|}{ Gracilariaceae } \\
\hline 99. Gracilaria blodgettii Harvey & 1,3 & $\mathrm{~W}, \mathrm{~S}$ & $\mathrm{M}, \mathrm{E}$ & $\mathrm{R}$ & $\mathrm{A}, \mathrm{B}$ & & 221212212222130 \\
\hline $\begin{array}{l}\text { 100. G. bursa-pastoris } \\
\text { (S.G. Gmelin) P.C. Silva }\end{array}$ & 3 & $\mathrm{R}$ & $\mathrm{E}$ & As, $\mathrm{Cm}$ & A & NRT & 22123 \\
\hline 101. G. caudata J. Agardh & $1,2,3,5$ & $\mathrm{~W}, \mathrm{R}$ & $\mathrm{M}, \mathrm{E}$ & $\mathrm{R}, \mathrm{Cm}$ & $\mathrm{A}, \mathrm{B}$ & & 2206822069 \\
\hline
\end{tabular}

SIMBOLS: Localities: 1. Sánchez Magallanes 2. La Machona 3. Laguna La Machona 4. Playa Paraíso 5. Laguna Mecoacan 6. San Pedro Centla Seasonality: W (Winter rains) S (Dry) R (Summer rains) Environment: M: Marine E: Estuarine References (Data source) A. This study B. Senties \& Dreckmann (2013) C. Quiroz-González, et al. (2017) OBS. (Observations) NRA: New record for Atlantic coast of Mexico NRT: New record for littoral of Tabasco Substrate: R: Rocky As: Artificial Substrate Cm: Cement floor Avp: Aquatic vascular plants Epi: Epiphytic on other algae 
Table 2. Continued...

\begin{tabular}{|c|c|c|c|c|c|c|c|}
\hline DIVISION/SPECIES & LOCALITIES & SEASONALITY & ENVIRONMENT & SUBSTRATE & REFERENCE & OBS. & $\begin{array}{c}\text { ENCB Herbarium } \\
\text { number }\end{array}$ \\
\hline 102. G. cervicornis (Turner) J. Agardh & 1 & $\mathrm{R}$ & M & $\mathrm{R}$ & $\mathrm{A}, \mathrm{B}$ & & 2211022112 \\
\hline 103. G. damaecornis J. Agardh & 1,2 & $\mathrm{R}$ & M & $\mathrm{R}$ & $\mathrm{A}, \mathrm{B}$ & & 2185221997 \\
\hline $\begin{array}{l}\text { 104. G. flabelliformis (P. Crouan \& } \\
\text { H. Crouan) Fredericq \& Gurgel }\end{array}$ & 1,3 & $\mathrm{~S}, \mathrm{R}$ & $\mathrm{M}, \mathrm{E}$ & $\mathrm{R}, \mathrm{Cm}$ & A & NRT & 218742187522109 \\
\hline $\begin{array}{l}\text { 105. Gracilariopsis longissima } \\
\text { (S.G. Gmelin) M. Steentoft, } \\
\text { L.M. Irvine \& W.F. Farnham }\end{array}$ & 1,3 & $\mathrm{~S}, \mathrm{R}$ & M,E & $\mathrm{R}, \mathrm{Cm}$ & A & NRT & 2208522089 \\
\hline \multicolumn{8}{|l|}{ Halymeniales } \\
\hline Halymeniaceae & & & & & & & 217662199521996 \\
\hline 106. Grateloupia filiformis Kützing & $1,2,3$ & $\mathrm{~W}, \mathrm{~S}, \mathrm{R}$ & M,E & $\mathrm{R}, \mathrm{Cm}$ & $\mathrm{A}, \mathrm{B}$ & & \\
\hline 107. G. gibbesii Harvey & 1 & $\mathrm{~S}$ & M & $\mathrm{R}$ & A & NRT & 2209422107 \\
\hline 108. G. subpectinata Holmes & 1.3 & $\mathrm{~W}, \mathrm{~S}, \mathrm{R}$ & $\mathrm{M}, \mathrm{E}$ & $\mathrm{R}$, As & A & NRA & 22002122031 \\
\hline 109. Prionitis pterocladina M.J. Wynne & 1 & $\mathrm{R}$ & M & $\mathrm{R}$ & A & NRT & 2206522067 \\
\hline \multicolumn{8}{|l|}{ Rhodymeniales } \\
\hline \multicolumn{8}{|l|}{ Lomentariaceae } \\
\hline $\begin{array}{l}\text { 110. Ceratodictyon variabile (J. Agardh) } \\
\text { R.E. Norris }\end{array}$ & 1,4 & $\mathrm{R}$ & M & $\mathrm{R}$ & A & NRT & 2208622088 \\
\hline \multicolumn{8}{|l|}{ OCHROPHYTA } \\
\hline \multicolumn{8}{|l|}{ Phaeophyceae } \\
\hline \multicolumn{8}{|l|}{ Dictyotales } \\
\hline \multicolumn{8}{|l|}{ Dictyotaceae } \\
\hline 111. Dictyopteris delicatula J.V. Lamouroux & 4 & $\mathrm{R}$ & M & R, Epi & A & NRT & 21996 \\
\hline 112. Dictyota crenulata J. Agardh & 4 & $\mathrm{~S}, \mathrm{R}$ & M & $\mathrm{R}$ & A & NRT & 2195822148 \\
\hline \multicolumn{8}{|l|}{ Asterocladales } \\
\hline \multicolumn{8}{|l|}{ Asterocladaceae } \\
\hline $\begin{array}{l}\text { 113. Asterocladon rhodochortonoides } \\
\text { (Børgesen) S. Uwai, C. Nagasato, } \\
\text { T. Motomura \& K. Kogame }\end{array}$ & 4 & $\mathrm{~S}$ & M & Epi & $\mathrm{A}$ & NRT & 2194722146 \\
\hline \multicolumn{8}{|l|}{ Scytothamnales } \\
\hline \multicolumn{8}{|l|}{ Asteronemataceae } \\
\hline $\begin{array}{l}\text { 114. Asteronema breviarticulatum } \\
\text { (J. Agardh) Ouriques \& Bouzon }\end{array}$ & 4 & $\mathrm{R}$ & M & $\mathrm{R}$ & A & NRT & 219592196021971 \\
\hline \multicolumn{8}{|l|}{ Bachelotiaceae } \\
\hline $\begin{array}{l}\text { 115. Bachelotia antillarum (Grunow) } \\
\text { Gerloff }\end{array}$ & 1 & $\mathrm{R}$ & M & $\mathrm{R}$ & A & NRT & 2193321954 \\
\hline \multicolumn{8}{|l|}{ Ectocarpales } \\
\hline \multicolumn{8}{|l|}{ Acinetosporaceae } \\
\hline $\begin{array}{l}\text { 116. Feldmannia duchassaingiana } \\
\text { (Grunow) Aisha \& Shameel }\end{array}$ & 1,4 & $\mathrm{R}$ & M & $\mathrm{R}$ & A & NRT & 22481 \\
\hline 117. F. irregularis (Kützing) Hamel & 1 & $\mathrm{R}$ & M & $\mathrm{R}$ & A & NRT & 22482 \\
\hline 118. F. mitchelliae (Harvey) H.S. Kim & $1,2,3,4$ & $\mathrm{~S}, \mathrm{R}$ & $\mathrm{M}, \mathrm{E}$ & Epi, Cm & A & NRT & $\begin{array}{c}219462194821949 \\
22144\end{array}$ \\
\hline $\begin{array}{l}\text { 119. Herponema tortugense (W.R. Taylor) } \\
\text { W.R. Taylor }\end{array}$ & 3 & S & $\mathrm{E}$ & As & $\mathrm{A}$ & NRT & TAB-16-B/01 \\
\hline \multicolumn{8}{|l|}{ Chordariaceae } \\
\hline $\begin{array}{l}\text { 120. Hecatonema floridanum (W.R. Taylor) } \\
\text { W.R. Taylor }\end{array}$ & 1 & $\mathrm{R}$ & M & Epi & A & NRT & 21853 \\
\hline $\begin{array}{l}\text { 121. Kuetzingiella elachistaeformis } \\
\text { (Heydrich) M. Balakrishnan \& Kinkar }\end{array}$ & 1,4 & $\mathrm{~S}, \mathrm{R}$ & M & Epi & A & NRT & 21935 \\
\hline \multicolumn{8}{|l|}{ Ectocarpaceae } \\
\hline $\begin{array}{l}\text { 122. Ectocarpus siliculosus (Dillwyn) } \\
\text { Lyngbye }\end{array}$ & $1,2,3,4$ & $\mathrm{~S}, \mathrm{R}$ & $\mathrm{M}, \mathrm{E}$ & $\mathrm{R}, \mathrm{Cm}$ & A & NRT & 219382194521950 \\
\hline \multicolumn{8}{|l|}{ Scytosiphonaceae } \\
\hline $\begin{array}{l}\text { 123. Chnoospora minima (Hering) } \\
\text { Papenfuss }\end{array}$ & $1,2,4$ & $\mathrm{~S}, \mathrm{R}$ & M & $\mathrm{R}$ & A & NRT & 219562196121970 \\
\hline
\end{tabular}

SIMBOLS: Localities: 1. Sánchez Magallanes 2. La Machona 3. Laguna La Machona 4. Playa Paraíso 5. Laguna Mecoacan 6. San Pedro Centla Seasonality: W (Winter rains) S (Dry) R (Summer rains) Environment: M: Marine E: Estuarine References (Data source) A. This study B. Senties \& Dreckmann (2013) C. Quiroz-González, et al. (2017) OBS. (Observations) NRA: New record for Atlantic coast of Mexico NRT: New record for littoral of Tabasco Substrate: R: Rocky As: Artificial Substrate Cm: Cement floor Avp: Aquatic vascular plants Epi: Epiphytic on other algae 
Table 2. Continued...

\begin{tabular}{|c|c|c|c|c|c|c|c|}
\hline DIVISION/SPECIES & LOCALITIES & SEASONALITY & ENVIRONMENT & SUBSTRATE & REFERENCE & OBS. & $\begin{array}{c}\text { ENCB Herbarium } \\
\text { number }\end{array}$ \\
\hline $\begin{array}{l}\text { 124. Colpomenia sinuosa } \\
\text { (Mertens ex Roth) Derbès \& Solier }\end{array}$ & $1,2,4$ & $\mathrm{~S}, \mathrm{R}$ & M & $\mathrm{R}$ & A & NRT & 2194222136 \\
\hline $\begin{array}{l}\text { 125. Rosenvingea floridana (W.R. Taylor) } \\
\text { W.R. Taylor }\end{array}$ & 1 & $\mathrm{~S}$ & M & $\mathrm{R}$ & A & NRT & 22143 \\
\hline 126. R. intricata (J. Agardh) Børgesen & 1 & $\mathrm{~S}, \mathrm{R}$ & $\mathrm{M}, \mathrm{E}$ & $\mathrm{R}$ & $\mathrm{A}, \mathrm{B}$ & & 22155 \\
\hline 127. R. orientalis (J. Agardh) Børgesen & 1 & $\mathrm{~S}$ & M & $\mathrm{R}$ & A & NRT & 22135 \\
\hline \multicolumn{8}{|l|}{ Sphacelariales } \\
\hline \multicolumn{8}{|l|}{ Sphacelariaceae } \\
\hline 128. Sphacelaria rigidula Kützing & $1,2,3$ & $\mathrm{~W}, \mathrm{R}$ & $\mathrm{M}, \mathrm{E}$ & Epi, Cm, As & A & NRT & 219142196521967 \\
\hline 129. S. tribuloides Meneghini & $1,2,3$ & $\mathrm{R}$ & $\mathrm{M}, \mathrm{E}$ & $\mathrm{R}, \mathrm{Cm}$ & A & NRT & 2195222150 \\
\hline \multicolumn{8}{|l|}{ CHLOROPHYTA } \\
\hline \multicolumn{8}{|l|}{ Ulvophyceae } \\
\hline \multicolumn{8}{|l|}{ Phaeophilales } \\
\hline \multicolumn{8}{|l|}{ Phaeophilaceae } \\
\hline $\begin{array}{l}\text { 130. Phaeophila dendroides (P.L. Crouan } \\
\text { \& H.M. Crouan) Batters }\end{array}$ & $1,2,4,5$ & $\mathrm{~W}, \mathrm{~S}, \mathrm{R}$ & $\mathrm{M}, \mathrm{E}$ & Epi & A & NRT & 21863 \\
\hline \multicolumn{8}{|l|}{ Ulvales } \\
\hline \multicolumn{8}{|l|}{ Kornmanniaceae } \\
\hline $\begin{array}{l}\text { 131. Blidingia marginata (J. Agardh) P.J.L. } \\
\text { Dangeard ex Bliding }\end{array}$ & 1,6 & W, S & M,E & Avp & $\mathrm{A}, \mathrm{C}$ & & 2192021944 \\
\hline 132. Bl. minima (Nägeli ex Kützing) Kylin & $1,4,5,6$ & W, S & $\mathrm{M}, \mathrm{E}$ & Epi, Avp & A & NRT & $\begin{array}{c}218782189621916 \\
21969\end{array}$ \\
\hline \multicolumn{8}{|l|}{ Ulvaceae } \\
\hline 133. Ulva clathrata (Roth) C. Agardh. & 3 & $\mathrm{~W}$ & $\mathrm{E}$ & As & A & NRT & 21864 \\
\hline 134. U. compressa Linnaeus & $1,3,4,5,6$ & $\mathrm{~W}, \mathrm{~S}, \mathrm{R}$ & $\mathrm{M}, \mathrm{E}$ & $\mathrm{R}, \mathrm{Epi}, \mathrm{Cm}$ & $\mathrm{A}, \mathrm{C}$ & & 21775 \\
\hline 135. U. fasciata Delile & 1,4 & $\mathrm{~S}, \mathrm{R}$ & M & $\mathrm{R}$ & $\mathrm{A}, \mathrm{C}$ & & 2184221894 \\
\hline 136. U. flexиosa Wulfen & $3,4,6$ & $\mathrm{~W}, \mathrm{~S}, \mathrm{R}$ & $\mathrm{M}, \mathrm{E}$ & $\mathrm{R}, \mathrm{As}$ & $\mathrm{A}, \mathrm{C}$ & & 217782184521882 \\
\hline $\begin{array}{l}\text { 137. U. flexuosa subsp. paradoxa } \\
\text { (C. Agardh) M.J. Wynne }\end{array}$ & 6 & W, S & $\mathrm{E}$ & $\mathrm{Cm}$ & $\mathrm{A}, \mathrm{C}$ & NRT & 2187321928 \\
\hline 138. U. intestinalis Linnaeus & $1,4,6$ & $\mathrm{~W}, \mathrm{~S}, \mathrm{R}$ & $\mathrm{M}, \mathrm{E}$ & $\mathrm{R}, \mathrm{Cm}$ & $\mathrm{A}, \mathrm{C}$ & & 2191521932 \\
\hline 139. U. rigida C. Agardh & 1,4 & $\mathrm{~W}, \mathrm{~S}, \mathrm{R}$ & M & $\mathrm{R}$ & $\mathrm{A}, \mathrm{C}$ & & 2185621868 \\
\hline \multicolumn{8}{|l|}{ Ulvellaceae } \\
\hline $\begin{array}{l}\text { 140. Ulvella lens P.L. Crouan \& H.M. } \\
\text { Crouan }\end{array}$ & 1,2 & $\mathrm{R}$ & M & Epi & A & NRT & 21770 \\
\hline $\begin{array}{l}\text { 141. Ul. scutata (Reinke) R. Nielsen, C.J. } \\
\text { O'Kelly \& B. Wysor }\end{array}$ & 3 & $\mathrm{~S}$ & $\mathrm{E}$ & As & A & NRT & TAB-ENCB/A \\
\hline $\begin{array}{l}\text { 142. Ul. viridis (Reinke) R. Nielsen, C.J. } \\
\text { O'Kelly \& B. Wysor }\end{array}$ & $1,4,5$ & $\mathrm{~W}, \mathrm{R}$ & $\mathrm{M}, \mathrm{E}$ & Epi & A & NRT & 2177022073 \\
\hline \multicolumn{8}{|l|}{ Boodleaceae } \\
\hline 143. Boodlea composita (Harvey) F. Brand & 4 & $\mathrm{~W}$ & M & $\mathrm{R}$ & A & NRT & 21901 \\
\hline $\begin{array}{l}\text { 144. Cladophoropsis membranacea } \\
\text { (Hofman Bang ex C. Agardh) Børgesen }\end{array}$ & 4 & $\mathrm{~S}$ & M & $\mathrm{R}$ & A & NRT & 21890 \\
\hline 145. C. sundanensis Reinbold & 1 & $\mathrm{R}$ & M & $\mathrm{R}$ & A & NRT & TAB-ENCB/B1 \\
\hline \multicolumn{8}{|l|}{ Cladophoraceae } \\
\hline $\begin{array}{l}\text { 146. Chaetomorpha aerea (Dillwyn) } \\
\text { Kützing }\end{array}$ & 1 & $\mathrm{~S}$ & M & $\mathrm{R}$ & A & NRT & 21914 \\
\hline 147. Ch. antennina (Bory) Kützing & $1,2,4$ & $\mathrm{~W}, \mathrm{~S}, \mathrm{R}$ & $\mathrm{M}$ & $\mathrm{R}$ & $\mathrm{A}, \mathrm{C}$ & & 2177121855 \\
\hline 148. Ch. linum (O.F. Müller) Kützing & 3.5 & W, S & $\mathrm{M}, \mathrm{E}$ & Epi, Avp & A & NRT & 2177721860 \\
\hline 149. Ch. minima Collins \& Hervey & 3 & $\mathrm{~S}$ & $\mathrm{E}$ & Epi & A & NRT & 21924 \\
\hline 150. Ch. nodosa Kützing & 1,4 & $\mathrm{~W}, \mathrm{R}$ & M & $\mathrm{R}$ & $\mathrm{A}, \mathrm{C}$ & & 2183921910 \\
\hline 151. Cladophora coelothrix Kützing & 1 & $\mathrm{R}$ & M & $\mathrm{R}$ & A & NRT & 21883 \\
\hline 152. Cl. crispula Vickers & 3,6 & $\mathrm{R}$ & $\mathrm{E}$ & As, $\mathrm{Cm}$ & A & NRT & 21937 \\
\hline 153. Cl. crystallina (Roth) Kützing & 1 & $\mathrm{R}$ & M & $\mathrm{R}$ & A & NRT & $\mathrm{TAB} / 15-54 / 04$ \\
\hline 154. Cl. dalmatica Kützing & 4 & $\mathrm{~S}$ & M & $\mathrm{R}$ & A & NRT & 21779 \\
\hline 155. Cl. flexuosa (O.F. Müller) Kützing & 1 & $\mathrm{R}$ & M & $\mathrm{R}$ & A & NRT & TAB-15-54/02 \\
\hline
\end{tabular}


Table 2. Continued...

\begin{tabular}{|c|c|c|c|c|c|c|c|}
\hline DIVISION/SPECIES & LOCALITIES & SEASONALITY & ENVIRONMENT & SUBSTRATE & REFERENCE & OBS. & $\begin{array}{c}\text { ENCB Herbarium } \\
\text { number }\end{array}$ \\
\hline $\begin{array}{l}\text { 156. Cl. fracta (O.F. Müller ex Vahl) } \\
\text { Kützing }\end{array}$ & 1 & $\mathrm{~S}$ & M & $\mathrm{R}$ & A & NRT & 21858 \\
\hline $\begin{array}{l}\text { 157. Cl. glomerata var. crassior (C. Agardh) } \\
\text { Hoek }\end{array}$ & 1 & $\mathrm{R}$ & M & $\mathrm{R}$ & A & NRT & TAB-16-A/01 \\
\hline 158. Cl. hutchinsiae (Dillwyn) Kützing & 1,4 & S & M & $\mathrm{R}$ & A & NRT & 22137 \\
\hline 159. Cl. sericea (Hudson) Kützing & $1,4,6$ & $\mathrm{~S}, \mathrm{R}$ & $\mathrm{M}, \mathrm{E}$ & $\mathrm{R}, \mathrm{Cm}$ & A & NRT & 218842188721891 \\
\hline 160. Cl. submarina P. Crouan \& H. Crouan & 1 & $\mathrm{R}$ & M & $\mathrm{Cm}$ & A & NRT & 21897 \\
\hline $\begin{array}{l}\text { 161. C. vagabunda (Linnaeus) van den } \\
\text { Hoek }\end{array}$ & $1,2,4,6$ & $\mathrm{~W}, \mathrm{~S}, \mathrm{R}$ & $\mathrm{M}, \mathrm{E}$ & $\mathrm{R}, \mathrm{Cm}$ & $\mathrm{A}, \mathrm{C}$ & & $\begin{array}{c}217702177221904 \\
21918\end{array}$ \\
\hline $\begin{array}{l}\text { 162. Pseudorhizoclonium africanum } \\
\text { (Kützing) Boedeker }\end{array}$ & $1,2,3$ & $\mathrm{~W}, \mathrm{~S}$ & $\mathrm{M}, \mathrm{E}$ & Epi, As & $\mathrm{A}$ & NRT & 218602188621926 \\
\hline 163. Rhizoclonium riparium (Roth) Harvey & $1,3,4,5,6$ & $\mathrm{~W}, \mathrm{~S}, \mathrm{R}$ & $\mathrm{M}, \mathrm{E}$ & Epi & $\mathrm{A}, \mathrm{C}$ & & 2184021861 \\
\hline $\begin{array}{l}\text { 164. Willeella brachyclados (Montagne) } \\
\text { M.J. Wynne }\end{array}$ & $1,5,6$ & $\mathrm{~W}, \mathrm{R}$ & $\mathrm{M}, \mathrm{E}$ & $\mathrm{R}, \mathrm{Cm}$ & A & NRT & 2177322311 \\
\hline \multicolumn{8}{|l|}{ Bryopsidales } \\
\hline \multicolumn{8}{|l|}{ Bryopsidaceae } \\
\hline 165. Bryopsis halliae W.R. Taylor & 4 & $\mathrm{R}$ & M & $\mathrm{R}$ & A & NRT & 21843 \\
\hline 166. B, hypnoides J.V. Lamouroux & 1,4 & $\mathrm{R}$ & M & $\mathrm{R}$ & A & NRT & 2189521919 \\
\hline 167. B. pennata (Kützing) Collins \& Hervey & 1,4 & $\mathrm{R}$ & M & $\mathrm{R}$ & A & NRT & 2184621877 \\
\hline $\begin{array}{l}\text { 168. B. pennata var. leprieurii (Kützing) } \\
\text { Collins \& Hervey }\end{array}$ & 1,4 & $\mathrm{R}$ & M & $\mathrm{R}$ & A & NRT & 2177621841 \\
\hline $\begin{array}{l}\text { 169. B. pennata var. secunda (Harvey) } \\
\text { Collins \& Hervey }\end{array}$ & 1 & $\mathrm{R}$ & M & $\mathrm{R}$ & A & NRT & 218472186322145 \\
\hline 170. B. plumosa (Hudson) C. Agardh. & 1,4 & $\mathrm{~S}, \mathrm{R}$ & M & $\mathrm{R}$ & A & NRT & 2177421844 \\
\hline $\begin{array}{l}\text { 171. Pseudobryopsis blomquistii } \\
\text { Díaz-Piferrer }\end{array}$ & 4 & $\mathrm{~S}$ & M & $\mathrm{R}$ & A & NRT & 22149 \\
\hline $\begin{array}{l}\text { 172. Trichosolen duchassaingii (J. Agardh) } \\
\text { W.R. Taylor }\end{array}$ & 4 & $\mathrm{R}$ & M & $\mathrm{R}$ & A & NRT & 2186621867 \\
\hline \multicolumn{8}{|l|}{ Ulotrichales } \\
\hline \multicolumn{8}{|l|}{ Ulotrichaceae } \\
\hline $\begin{array}{l}\text { 173. Urospora penicilliformis (Roth) } \\
\text { Areschoug }\end{array}$ & 3 & W & $\mathrm{E}$ & Avp & A & NRT & 21848 \\
\hline
\end{tabular}

This species has been reported from Korea, Vietnam, Japan, China, India, Australia, New Zealand, Hawaii, Mariana Islands, Solomon Islands and Galapagos Islands (Guiry \& Guiry, 2017). Therefore this is the first record of Gayliella fimbriata for Tabasco and moreover, for the Atlantic coast of America.

Grateloupia subpectinata Holmes (Figures $3 \mathrm{~d}-\mathrm{j}$ )

Type locality: Japan.

Heterotypic synonyms:

Grateloupia luxurians (A. Gepp \& E.S. Gepp) R.J. Wilkes, L.M. McIvor \& Guiry 2005: 58

Grateloupia filicina var. luxurians A. Gepp \& E.S. Gepp 1906: 259

Representative specimens examined: Sánchez Magallanes Breakwater (Mateo Cid, Mendoza González \& Valencia Torres, 10.iv.2016, ENCB 22996, female thalli and tetrasporangial thalli); Laguna La Machona (Mateo Cid \& Mendoza González, 10.iv.2016, ENCB 22006, female).

Habit and anatomy: The thallus arising from a discoid holdfast is formed by simple or pinnate erect fronds tapering at the ends, mainly compressed, mucilaginous but firm, reddish to blackish purple, sometimes greenish. Thalli are $2.0-7.0 \mathrm{~cm}$ high, simple linear compressed and $375-450 \mu \mathrm{m}$ diameter; cortex 3-5 layers, the cells moniliform or rounded to stellate in

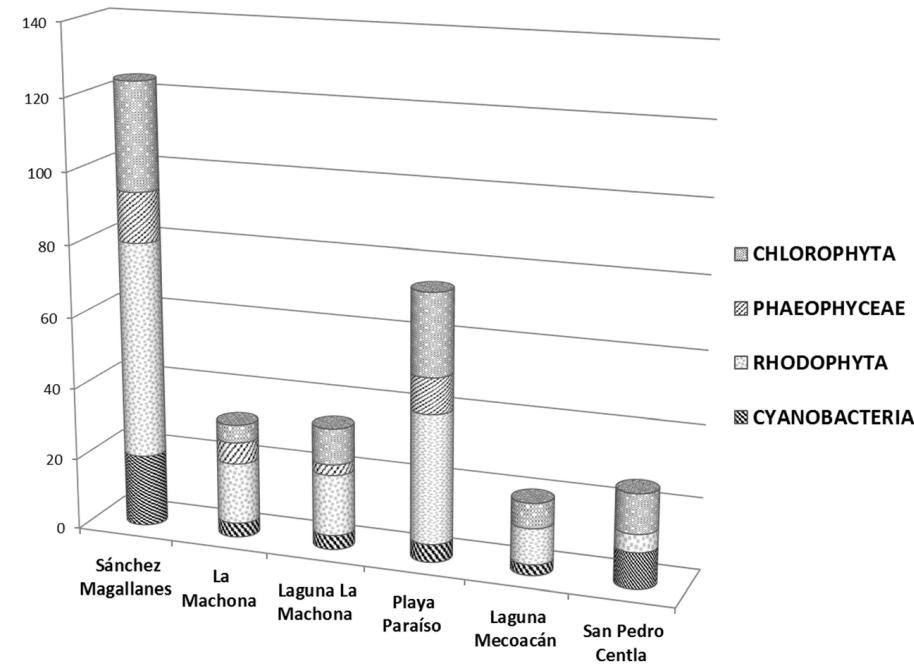

Figure 2. Number of species per division per locality 

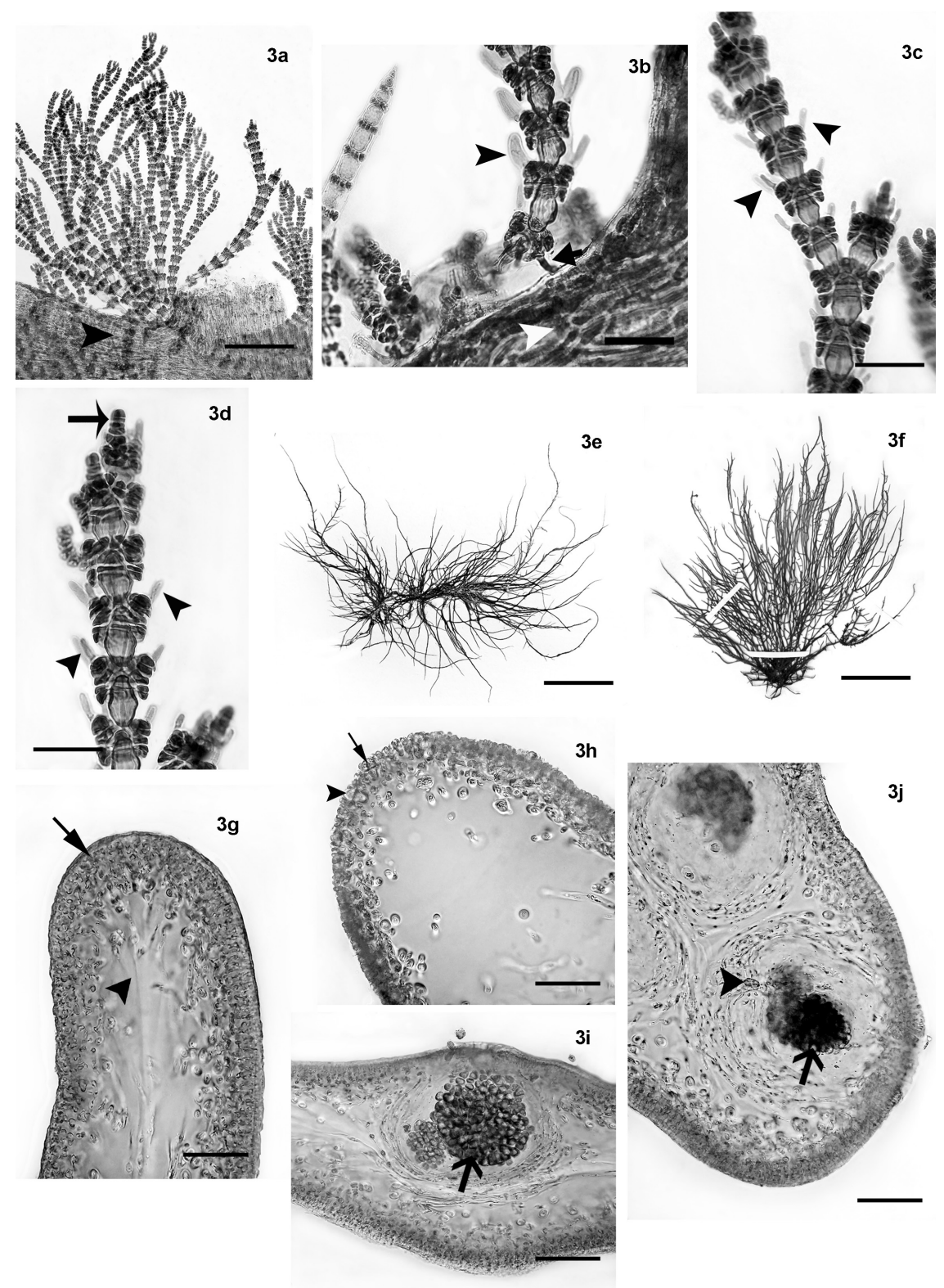

Figure 3. Gayliella fimbriata: Sánchez Magallanes (ENCB 22091). 3a. General appearance of the filamentous thallus growing on Spyridia sp (arrowhead). Scale 200 $\mu \mathrm{m}$. 3b: Base of the thallus on the host (white arrowhead). Note rhizoid (arrow), and the prominent gland cells (black arrowhead) on the cortical bands. Scale $135 \mu \mathrm{m}$. 3c: Detail of verticilar arrangement of gland cells (arrowheads). Scale $25 \mu \mathrm{m}$. 3d: Detail of an apical branch without forcipates apices. Note the apical cell (arrow) and gland cells (arrowheads). Scale $20 \mu \mathrm{m}$. 3e. Grateloupia subpectinata: Sanchez Magallanes (ENCB 22031), a: tetrasporangial thallus. Scale 1.6 cm. $3 \mathrm{f}$ : G. subpectinata: Sanchez Magallanes (ENCB 22016), Female thallus. Scale $1.8 \mathrm{~cm} .3 \mathrm{~g}$ : Cross-section through a blade showing cortex (arrow) and developing medulla. (Arrowhead). Scale $150 \mu \mathrm{m} .3 \mathrm{~h}$ : Cross-section through a blade showing an immature tetrasporangium (arrowhead) and a mature, cruciately divided tetrasporangium. (Arrow) Scale $95 \mu \mathrm{m}$. 3i: Close-up of mature gonimoblast (arrow). Scale $80 \mu \mathrm{m}$. 3j: Cross-section through a blade showing the gonimoblasts (arrow) borne on an expanded fusion cell. Note the elongated ampullar filaments (arrowhead). Scale $60 \mu \mathrm{m}$.

shape 7-8 $\mu \mathrm{m} \times 7.5-8.5 \mu \mathrm{m}$; medulla filamentous with lax filaments of 3-4 $\mu \mathrm{m}$ in diameter.

Tetrasporophytes and gametophytes isomorphic, the gametophytes dioecious with reproductive structures scattered over the entire thallus except the basal parts.

Tetrasporangia initiated from inner cortical cells, cruciately divided when mature, 14-15 $\mu \mathrm{m}$ wide x 29-30 $\mu \mathrm{m}$ long. Carposporangial ampullae not found; gonimoblasts $60-80 \mu \mathrm{m}$ immersed inside the medulla and surrounded by branched ampullar filaments and a network of secondary medullary filaments.

Comments: Regarding the dimensions of the thallus, tetrasporangia and gonimoblasts show a smaller size than those recorded in specimens from Japan (Faye et al., 2004). Mexican specimens of Grateloupia subpectinata are morphologically similar to G. filicina (J.V. Lamouroux) J. Agardh. Nevertheless, both species have a considerable morphological plasticity (Faye et al., 2004, Verlaque et al., 2005). Compressed and branching Grateloupia species are notoriously difficult to identify due to their morphological similarity and lack of clear-cut diagnostic characters. The characteristics of our specimens are closer to the concept of Grateloupia subpectinata, so it is considered a new record for Tabasco and the Mexican Atlantic. This taxon has been also recorded in the Caribbean Islands (Wynne 2017), Britain, France, Spain and Australia (Guiry \& Guiry 2017). 


\section{Discussion}

\section{Floristic composition}

Here we report the presence of 115 previously unreported benthic marine and estuarine algae and 26 Cyanobacteria for the continental coasts of Tabasco, two of them, new records for the Atlantic coast of Mexico. Including the recently published reports of species from Tabasco (Senties \& Dreckmann 2013, Quiroz-González et al., 2017), the total number of species increases to 194 . This represents an increase of $150 \%$ (67) updated from the number published in the recent articles of Senties \& Dreckmann (2013) and Quiroz-González et al. (2017), who based their analyses on reports in the literature and recent collections obtained by these authors. When analyzing the different taxonomic groups (Table 2), our survey resulted in a considerable increase in the number of Rhodophyta taxa (53.9\%), followed by Chlorophyta (29.5\%), and Phaeophyceae (16.6\%).

Among the new records we can highlight: Acrochaetium sagreanum, A. microscopicum, Antithamnionella boergesenii, An. elegans, Heterosiphonia crispella, Gayliella mazoyerae, G. fimbriata, Peyssonnelia armorica, Asterocladon rhodochortonoides, Hecatonema floridanum, Herponema tortugense, Phaeophila dendroides, Ulvella lens, Ul. scutata, and Ul. viridis. Epiphytes are a very important group in the algal flora, especially in Tabasco, whose coast has many floodplains, extensive sandy beaches, coastal lagoons, breakwaters, and very exposed rocky coasts, lacking of algae.

In this study, a total of 26 taxa of Cyanobacteria were found at six localities of Tabasco and constituted $17.67 \%$ of the new records. A list of identified species is given in Table 2. There was a dominance of members of the orders Oscillatoriales and Pleurocapsales (27\%), and Nostocales (15\%), and all 26 taxa were reported for the first time in studies of the Tabasco coast. Species with wide distribution in the study area are Lyngbya confervoides, Calothrix parietina and Scytonematopsis crustacea, all of which have been reported in Campeche and Quintana Roo. With regard to the species of Chroococcales and Pleurocapsales, such as Pseudocapsa maritima, Chroococcus ercegovicii, Aphanocapsa littoralis, all were recorded from one locality, while Entophysalis conferta was present in five of the six study sites, being the most frequent and abundant of all the cyanobacteria recorded in our study.

In the last 50 years, the presence of cyanobacteria in brackishwater systems has been increasingly reported worldwide. Then, a wide diversity of cyanobacteria species can be found in brackish water systems (Lopes \& Vasconcelos 2011). In this study, ten species of Cyanobacteria were observed in San Pedro Centla, locality with estuarine environment, while in the Sanchez Magallanes breakwater 16 species were recorded. Finding such a number of species confirms the ability of cyanobacteria to colonize the substrate available, ability for epiphytism, and to form mats in brackish (estuarine) and marine waters.

Most of these organisms requires culture studies and, when possible also the application of molecular-genetic techniques (Bernecker \& Wehrtmann 2009). A detailed taxonomic analysis of these taxa will result in higher species numbers for Tabasco.

\section{Type of environment and substrate}

According to our study, of the 173 species, 109 develop exclusively in marine environment, 20 in estuarine environment, while 43 were located in both estuarine and marine environments. Most of the Rhodophyta develop in marine rocky areas, whereas members of Chlorophyta are established and developed in both marine and estuarine environments. In the case of brown algae, most of them (14) occur in the marine environment. These results agree with those described by McGlathery et al. (2013), who indicated that brown and red algae are almost exclusively marine species and the Chlorophyta can occasionally be abundant in the lower salt marsh zone in estuaries.

In our study, the localities with rocky substrate with marine water influence present high species richness compared with estuarine localities. A total of 89 species was registered in this type of substrate. The rocky substrate is favorable for the development of a great number of marine tropical seaweeds of Ceramiales, Corallinales, Cladophorales and Bryopsidales. On the other hand, the sampling sites with artificial substrate as plastic, net ropes, fishing lines, textiles and concrete debris present lower richness (25); it may be because the artificial substrate limits growth of marine algae and cyanobacteria because they aren't hard and/or stable (Santelices 1977, Garduño-Solórzano et al. 2005, Mateo-Cid et al. 2013). Moreover, our results agree with the statement by Hartog (1967), who indicates that the flora of the brackish water is poor in species in comparison with those of the sea and the fresh water. Most of the marine organisms are stenohaline and are unable to live permanently in water with a higher or lower salt-content than that of the sea.

\section{Epiphytic species}

Epiphytes are small size, often microscopic, and their adaptive strategy as epiphytes depends on the permanence of their host and is affected by competition between them for the substrate and light, among other factors. The identification of these species is complicated because their characteristics are difficult to observe. On the other hand, previous works were developed with different objectives, so they have not been similarly inventoried.

Seventy-five epiphytic species (Table 2) were found, 25 of which correspond to Cyanobacteria, 35 to Rhodophyta, six to Phaeophyceae and nine to Chlorophyta. The highest number of epiphytes was observed in the dry season with 45 , with a greater number of cyanobacterial species, as well as the families Acrochaetiaceae, Rhodomelaceae and Ceramiaceae, which cover other algae up to almost $90 \%$ of their surface. This finding agrees with what is observed in some brown algae and marine phanerogams, in which the level of epiphytism is greater when the surface and biomass of these organisms are larger. This condition is also attributed to the decrease of defense substances against the epiphytes as the host becomes senescent (Ortuño-Aguirre and Riosmena-Rodríguez, 2007).

Most of the epiphytes were frequently found growing on perennial red algae such as Acanthophora, Bryocladia, Gracilaria, and Grateloupia. It has been suggested that host longevity should be long enough to allow these organisms to complete their life cycle and that this might be a likely reason for the absence of epiphytes on annual and ephemeral algae (Santelices, 1977).

\section{Seasonality}

Temperature and light determine the latitudinal distribution of seaweeds, and therefore their geographical distribution for they also influence the composition, variation and periodicity of populations at the intertidal and subtidal levels (Santelices, 1977). In our study area summer days are 4 to 5 hours longer that in the winter. Hence, the availability of light for seaweed and estuarine organisms is higher, which influences the species richness of these organisms during the summer (rainy season), with 62 Rhodophyta (49.2\%), 15 Phaeophyceae (11.9\%), 28 Chlorophyta (22.2\%) and 21 Cyanobacteria (16.68\%). The lowest specific richness occurred in winter rains ("nortes" in which the rain is greater than $10.2 \%$ and temperature values between 20 and $22^{\circ} \mathrm{C}$ ) with 29 Rhodophyta (46.77\%), 1 Phaeophyceae (1.62\%), 19 Chlorophyta (30.65\%), and 13 Cyanobacteria (20.96\%). Finally, during the dry season 41 Rhodophyta (49.4\%), 11 Phaeophyceae (13.3\%), 24 Chlorophyta (28.9\%), and 7 Cyanobacteria (8.4\%) occurred (Figure 4) for our results are similar to those reported in previous studies in the Mexican Atlantic where mentioned that the species richness of algae is higher in the rainy season (Ortega et al., 2001, Callejas-Jimenez et al, 2005, Mateo-Cid et al., 2013). 


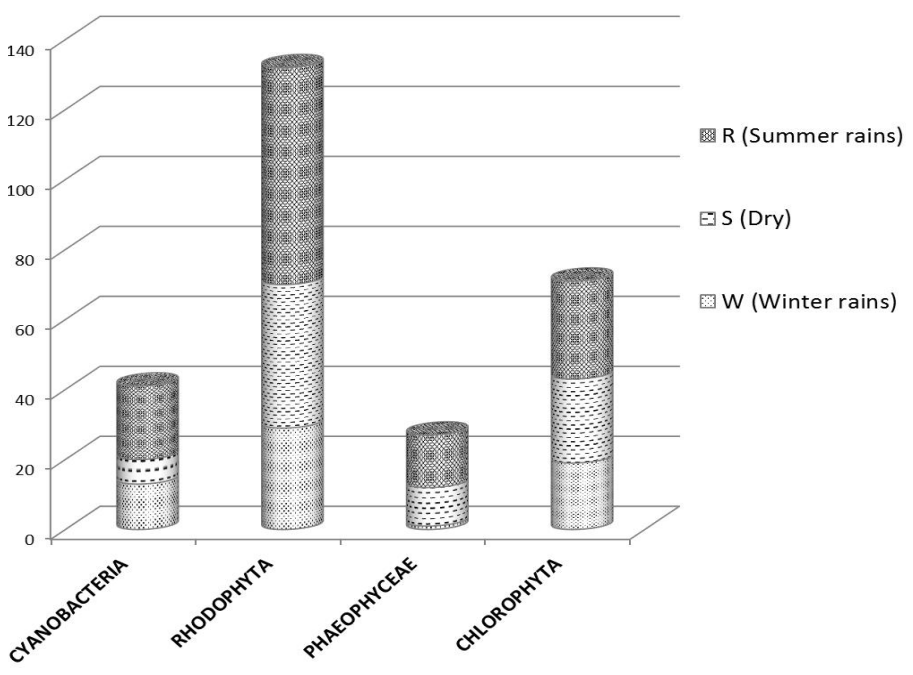

Figure 4. Number of species per division per season

Table 3. Feldmann and Cheney indexes for some marine and estuarine environments of the Gulf of Mexico and Mexican Caribbean coast.

\begin{tabular}{cccc}
\hline \multirow{2}{*}{ Locality } & Feldmann Index & & Cheney Index \\
\cline { 2 - 2 } & $\mathbf{( R / P )}$ & $(\mathbf{R}+\mathbf{C}) / \mathbf{P}$ \\
\hline Isla Mujeres, Quintana Roo & 4.50 & 7.15 \\
Isla Cozumel, Quintana Roo & 4.40 & 7.04 \\
Puerto Morelos, Quintana Roo & 3.40 & 5.60 \\
Coast of Yucatan & 6.00 & 9.00 \\
Coast of Campeche & 5.37 & 9.30 \\
Coast of Tabasco (This study) & 4.42 & 6.73 \\
\hline
\end{tabular}

In the rocky zone of the Sanchez Magallanes breakwater and Playa Paraiso breakwater numerous species of the families Corallinaceae, Rhodomelaceae, Ectocarpaceae, Dictyotaceae and Ulvaceae occurred. Rhodophyta dominates in number, with 60 in Sánchez Magallanes and 36 in Playa Paraíso. In the dry and rainy seasons Ulva fasciata, U. rigida, Cladophora vagabunda and Bryopsis pennata are common; while Asteronema breviarticulatum and Ectocarpus siliculosus are the most common in the rainy season. It is evident that in the estuarine areas the smallest number of species is presented, the Laguna Mecoacan having the lowest diversity with only 10 Rhodophyta, 7 Chlorophyta and 3 Cyanobacteria.

\section{Biogeography}

The results of the present study were analyzed applying the Feldmann and the Cheney indexes and compared with findings by Mateo-Cid \& Mendoza-González (2007) for Cozumel island; Mendoza-González et al. (2007) of Mujeres Island, Dreckmann et al. (1996) for Puerto Morelos, Huerta-Múzquiz et al. (1987), Ortegón-Aznar et al. (2001, 2009) and Sánchez-Molina et al. (2007) for the coast of Yucatán and Mateo-Cid et al. (2013) for the coast of Campeche. Table 3 shows the data obtained applying the Feldmann and Cheney indexes to the five regions mentioned; the phycoflora of the study area is similar to that of the coast of the state of Quintana Roo, with a value close to that obtained for the Cozumel and Mujeres Islands, both distinctly Caribbean localities.

The indexes used indicate that the marine and estuarine algae of the coast of Tabasco have a predominantly tropical distribution, with tropical elements such as Melanothamnus ferulaceus, Hypnea musciformis, Jania adhaerens, Gracilaria flabelliformis, Gracilariopsis longissima, Dictyopteris delicatula, Dictyota crenulata, Chnoospora minima, Chaetomorpha antennina, Ulva rigida and Cladophora flexuosa, among others. The phycoflora of the coast of Tabasco conforms to the pattern found in all coastlines of the states of the Gulf of Mexico (Ortega et al. 2001, Mateo-Cid et al., 2013).

\section{Acknowledgments}

The authors acknowledge the Instituto Politécnico Nacional (SIP-20161052, 20164274, 20170696, 20170767), which provided financial assistance, facilities and equipment necessary for the development of this study. The first two authors thank the Instituto Politécnico Nacional and the Comisión de Operación y Fomento de Actividades Académicas del I.P.N. for the fellowships granted ACMG and LEMC thank EDI/IPN.

\section{Author contributions}

A. Catalina Mendoza-González collected and identified specimens, analyzed data and participated in writing the text.

Luz Elena Mateo-Cid collected and identified specimens, participated in writing the text, analyzed data and edited the photographs.

Deisy Yazmín García-López collected and identified the samples and edited map.

All authors contributed to critical revision, adding intellectual content.

\section{Conflicts of interest}

The authors declare that they have no conflict of interest related to the publication of this manuscript.

\section{References}

ANAGNOSTIDIS, K. \& KOMÁREK, J. 1988. Modern approach to the classification system of Cyanophytes. 3. Oscillatoriales. Arch Hydrobiol Suppl. 80: 327-472.

BERNECKER, A. \& WEHRTMANN, I.S. 2009. New records of benthic marine algae and Cyanobacteria for Costa Rica, and a comparison with other Central American countries. Helgoland Mar. Res. 63:219-229.

CALLEJAS-JIMÉNEZ, M. E., SENTÍES, A. \& DRECKMANN, K.M. 2005. Macroalgas de Puerto Real, Faro Santa Rosalía y Playa Preciosa, Campeche, México, con algunas consideraciones florísticas y ecológicas para el estado. Hidrobiológica 15 (1): 89-96.

CHENEY, D. P. 1977. A new improved ratio for comparing seaweed floras. J Phycol. 13(suppl.): 1-13.

CHO, T. O., BOO, S. M., HOMMERSAND, M.H., MAGGS, C.A., MCIVOR, L. \& FREDERICQ, S. 2008. Gayliella gen. nov. in the tribe Ceramieae (Ceramiaceae, Rhodophyta) based on molecular and morphological evidence. J Phycol. 44(3): 721-738.

DAWES, C. J. \& MATHIESON, A.C. 2008. The seaweeds of Florida. University Press of Florida. Gainesville.

DRECKMANN, K. M., STOUT, I. \& SENTÍES, A. 1996. Lista actualizada de las algas marinas bentónicas de Puerto Morelos, Quintana Roo, Caribe mexicano. Polibotánica 3: 1-17.

DRECKMANN, K.M. \& DE LARA-ISASSI, G. 2000. Gracilaria caudata J. Agardh (Gracilariaceae, Rhodophyta) en el Atlántico mexicano. Hidrobiológica 10: $125-130$.

FAYE E.J., WANG, H.W., KAWAGUCHI, S., SHIMADA, S. \& MASUDA, M. 2004. Reinstatement of Grateloupia subpectinata (Rhodophyta, Halymeniaceae) based on morphology and $r b c$ L sequences. Phycol Res 52:59-67

FELDMANN, J. 1937. Recherches sur la végétation marine de la Méditerranée. La Côte des Albères. Rev Algol 10: 1-339.

FREDERICQ, S., GURGEL, C.F.D., NORRIS, J.N., EARLE, S., MATEO-CID, L.E., MENDOZA-GONZÁLEZ, A.C. \& SUÁREZ, A.M. 2009. Seaweeds (Rhodophyta, Chlorophyta and Phaeophyceae) of the Gulf of Mexico. In: Gulf of Mexico, origins Waters and Biota: Biodiversity. (D.L. Felder \& D.A. Camp, eds.). Texas A\&M University Press, College Station, Texas. v. 1, p. 45-87. 
GARCIA, E. \& VIDAL-ZEPEDA R. 1990a. Temperatura media. Atlas Nacional de México, Hoja IV.4.4, Instituto de Geografía, UNAM, México.

GARCÍA, E. \& VIDAL-ZEPEDA R. 1990b. Temperaturas extremas, Atlas Nacional de México, Hoja IV.4.5, Instituto de Geografía, UNAM, México.

GARDUÑO-SOLÓRZANO G., GODÍNEZ-ORTEGA, J. \& ORTEGA, M 2005. Distribución geográfica y afinidad por el sustrato de las algas verdes (Chlorophyceae) bentónicas de las costas mexicanas del Golfo de México y Mar Caribe. Bol Soc Bot Mex 76: 61-78.

GEPP, A. \& GEPP, E.S. 1906. Some marine algae from New South Wales. J Bot 44: $249-261$

GOMONT, M. 1892 '1893’. Monographie des Oscillariées (Nostocacées Homocystées). Deuxième partie. - Lyngbyées. Ann Sci Nat Bot, Ser 7 16: 91-264.

GUIRY, M. D. \& GUIRY, G.M. 2017. World-wide electronic Publication. National University of Ireland. Galway, Ireland. http://www. algaebase.org. (last access in $25 / 03 / 2017$ ).

GURGEL, C.F.D. \& FREDERICQ, S. 2004. Systematics of the Gracilariaceae (Gracilariales, Rhodophyta): a critical assessment based on $r b c \mathrm{~L}$ sequence analysis. J Phycol 40: 138-159.

HARTOG, C. 1967. Brackish water as an environment for Algae. Blumea 15: 31-43. HERNÁNDEZ-SANTANA, J.R., ORTIZ-PÉREZ, M.A, MÉNDEZ LINARES, A.P. \& GAMA CAMPILLO, L. 2008. Morfodinámica de la línea de costa del estado de Tabasco, México: tendencias desde la segunda mitad del siglo XX hasta el presente. Investigaciones Geográficas, Boletín del Instituto de Geografia UNAM 65: 7-21.

HUERTA-MÚZQUIZ, L., MENDOZA-GONZÁLEZ, A.C. \& MATEO-CID, L.E. 1987. Avance sobre un estudio de las algas marinas de la Península de Yucatán. Phytologia 62: 23-53.

KOMÁREK, J. 2010. Recent changes (2008) in cyanobacteria taxonomy based on a combination of molecular background with phenotype and ecological consequences (genus and species concept). Hydrobiologia 639: 245-259.

KOMÁREK, J. \& ANAGNOSTIDIS, K. 1995. Nomenclatural novelties in chroococcalean cyanoprokaryotes. Preslia 67: 15-23.

KOMÁREK, J., KASTOVSKY, J., MARES, J. \& JOHANSEN, J.R. 2014. Taxonomic classification of cyanoprokaryotes (cyanobacterial genera) 2014, using a polyphasic approach. Preslia 86: 295-335.

LITTLER, D.S. \& LITTLER, M.M. 2000. Caribbean reef plants. An identification guide to the reef plants of the Caribbean, Bahamas, Florida and Gulf of Mexico. Offshore Graphics. Washington.

LOPES, V.R. \& VASCONCELOS, M.V. 2011. Planktonic and benthic cyanobacteria of European brackish waters: a perspective on estuaries and brackish seas. Eur J Phycol 46:3, 292-304.

MCGLATHERY, K.J., SUNDBACK.K. \& FONG, P. 2013. Estuarine benthic algae. In Estuarine Ecology (J. Day Jr, B.C. Crump, W.M. Kemp \& A. YañezArancibia, eds.). New Jersey: A John Wiley \& Sons, Inc., Publication. New Jersey. p. 235-264

MATEO-CID, L.E. \& MENDOZA-GONZÁLEZ, A.C. 2007. Flora ficológica: Diversidad, importancia económica y conservación. In Biodiversidad acuática de la Isla Cozumel (L. Mejía-Ortiz, ed.) Distrito Federal: Plaza Valdés. p. 81-113.

MATEO-CID, L.E., MENDOZA-GONZÁLEZ, A.C. AVILA-ORTIZ, A.G. \& DÍAZ-MARTÍNEZ, S. 2013. Algas marinas bentónicas del litoral de Campeche, México. Acta Bot Mex 104: 53-92.

MENDOZA-GONZÁLEZ, A. C., MATEO-CID, L. E. \& SEARLES, R.B. 2007. Yucatán seaweeds from the offshore waters of Isla Mujeres, Quintana Roo, México. Bot Mar 50: 280-287.
OROZCO-VEGA, H. \& DRECKMANN, K.M. 1995. Macroalgas estuarinas del litoral Mexicano del Golfo de México. Cryptogamie Algol 16: 189-198.

ORTEGA, M. M., GODÍNEZ-ORTEGA, J. L. \& GARDUÑO-SOLÓRZANO, G. 2001. Catálogo de algas bénticas de las costas mexicanas del Golfo de México y Mar Caribe. Comisión Nacional para el Estudio de la Biodiversidad y Universidad Nacional Autónoma de México. México, D.F., México.

ORTEGÓN-AZNAR, I., GONZÁLEZ-GONZÁLEZ, J. \& SENTÍES, A. 2001. Estudio ficoflorístico de la laguna de Río Lagartos, Yucatán, México. Hidrobiológica 11(2): 97-104.

ORTEGÓN-AZNAR, I., SÁNCHEZ-MOLINA, I. \& CASANOVA-CETZ, R.A 2009. The distribution of marine algae in a coastal lagoon, northern Yucatan Peninsula, Mexico. Neotrop Biol Conserv 4(2): 99-105.

ORTUÑO-AGUIRRE, C. \& RIOSMENA-RODRÍGUEZ, R. 2007. Dinámica en el epifitismo de Padina concrescens (Dictyotales, Phaeophyta) en el sureste de la Península de Baja California, México. Cien Mar 33(3): 311-317.

QUIROZ-GONZÁLEZ, N., LEÓN-ÁLVAREZ, D. \& RIVAS-ACUÑA, M.G. 2017. Nuevos registros de algas verdes marinas (Ulvophyceae) para Tabasco, México. Acta Bot Mex 118: 121-138. DOI: http://dx.doi.org/10.21829/abm118. 2017.1204

RAMÍREZ, L.A. 1996. Estudio preliminar de las algas rojas (Rhodophyta) de litoral del estado de Tabasco, México. Tesis Profesional, Universidad Nacional Autónoma de México, Iztacala. Tlalnepantla, Estado México, México.

SÁNCHEZ-MOLINA, I., GONZÁLEZ-CEBALLOS, J., ZETINA-MOGUEL, C. \& CASANOVA-CETZ, R. 2007. Análisis de la biodiversidad de algas marinas situadas entre Uaymitún y Chuburná, Yucatán. Ingeniería 11(1): 43-51.

SANTELICES, B. 1977. Ecología de algas marinas bentónicas. Efectos de factores ambientales. Santiago de Chile: Pontificia Universidad Católica de Chile.

SCHNEIDER, C. W. \& SEARLES, R. B. 1991. Seaweeds of the southeastern United States. Cape Hatteras to Cape Cañaveral. Duke University Press. Durham.

SENTIES, A. \& DRECKMANN, K.M. 2013. Lista actualizada de las macroalgas de Tabasco, México. Acta Bot Ven 36 (2): 109-117.

SETCHELL, W.A. \& GARDNER, N.L. 1924. New marine algae from the Gulf of California. Proc Calif Acad Sci Series 4 12: 695-949, 77 plates.

TAYLOR, W. R. 1960. Marine algae of the eastern tropical and subtropical coasts of the America. University of Michigan Press. Ann Arbor

VERLAQUE, M., BRANNOCK, P.M., KOMATSU, T., VILLALARDBOHNSACK, M. \& MARSTON, M. 2005. The genus Grateloupia C. Agardh (Halymeniaceae, Rhodophyta) in the Thau Lagoon (France, Mediterranean): a case study of marine plurispecific introductions. Phycologia 44 (5): 477-496.

WON, B.Y., CHO, T.O. \& FREDERICQ, S. 2009. Morphological and molecular characterization of species of the genus Centroceras (Ceramiaceae, Ceramiales), including two new species. J Phycol 45: 227-250.

WILKES, R.J., MCIVOR, L.M. \& GUIRY, M.D. 2005. Using $r b c$ L sequence data to reassess the taxonomic position of some Grateloupia and Dermocorynus species (Halymeniaceae, Rhodophyta) from the north-eastern Atlantic. Eur J Phycol 40: 53-60.

WYNNE, M. J. 2017. A checklist of benthic marine algae of the tropical and subtropical western Atlantic: fourth revision. Nova Hedwigia Beihefte 145: 1-202.

Received: $27 / 05 / 2017$

Revised: 24/09/2017

Accepted: 25/09/2017

Published online: 26/10/2017 